Article

\title{
Optimization-Based Formulations for Short-Circuit Studies with Inverter-Interfaced Generation in PowerModelsProtection.j1
}

\author{
Arthur K. Barnes * ${ }^{D}$, Jose E. Tabarez $(D)$, Adam Mate $(D)$ and Russell W. Bent (D) \\ Los Alamos National Laboratory, Los Alamos, NM 87545, USA; jtabarez@lanl.gov (J.E.T.); amate@lanl.gov (A.M.); \\ rbent@lanl.gov (R.W.B.) \\ * Correspondence: abarnes@lanl.gov
}

Citation: Barnes, A.K.; Tabarez, J.E.; Mate, A.; Bent, R.W. Optimization-

Based Formulations for Short-Circuit Studies with Inverter-Interfaced Generation in

PowerModelsProtection.jl. Energies 2021, 14, 2160. https://doi.org/

$10.3390 /$ en14082160

Academic Editor: Ali Bidram

Received: 25 January 2021

Accepted: 7 April 2021

Published: 13 April 2021

Publisher's Note: MDPI stays neutral with regard to jurisdictional claims in published maps and institutional affiliations.

Copyright: (c) 2021 by the authors. Licensee MDPI, Basel, Switzerland. This article is an open access article distributed under the terms and conditions of the Creative Commons Attribution (CC BY) license (https:// creativecommons.org/licenses/by/ $4.0 /)$.

\begin{abstract}
Protecting inverter-interfaced microgrids is challenging as conventional time-overcurrent protection becomes unusable due to the lack of fault current. There is a great need for novel protective relaying methods that enable the application of protection coordination on microgrids, thereby allowing for microgrids with larger areas and numbers of loads while not compromising reliable power delivery. Tools for modeling and analyzing such microgrids under fault conditions are necessary in order to help design such protective relaying and operate microgrids in a configuration that can be protected, though there is currently a lack of tools applicable to inverter-interfaced microgrids. This paper introduces the concept of applying an optimization problem formulation to the topic of inverter-interfaced microgrid fault modeling, and discusses how it can be employed both for simulating short-circuits and as a set of constraints for optimal microgrid operation to ensure protective device coordination.
\end{abstract}

Keywords: power system operation; protective relaying; optimization; microgrid; distributed energy resources; distribution network; protection

\section{Introduction}

Protecting distribution networks is critically important. Protective devices isolate faults, prevent damage to sensitive equipment, and maintain continuous service to as much of the system as possible. Reconfiguring a distribution network to support networked microgrids [1] leads to scenarios where protection schemes may no longer achieve these desired outcomes. Developing adequate schemes is challenging due to blinding of protection, defeat of fuse-saving, sympathetic tripping of protection, and fault current falling below or above coordination limits. Such issues can result in the inability of conventional protection to operate or a loss of protection coordination [2,3].

Issues with protecting inverter-interfaced microgrids were recently investigated and evaluated by McDermott et al. [3]. A number of underlying difficulties were highlighted: the lack of fault current from inverter-interfaced generation [4], the varying fault current when transitioning from grid-connected to islanded mode [4], and the potential for normally meshed operation [5] and unbalanced operation due to single-phase loads [5]. Potential solutions, some with severe limitations, have been proposed for these challenges over the years [6].

Dewadasa et al. [5,7] introduced admittance protection for load protection. Kar et al. [8] investigated differential protection, based on the discrete S-transform, for line protection. Singh et al. [9] introduced an adaptive protection-coordination scheme that continuously monitors the state of the network. Pavlatos et al. [10] proposed a linguistic representation of waveforms, which was used to implement an efficient protective relay against faults. Jamali et al. [11] proposed a protection technique for networks with synchronous distributed generation units that does not require any communications infrastructures. 
Barnes et al. [12] investigated admittance relaying and [13] dynamic state estimation for the protection of microgrids. Methods and techniques, however, must be evaluated in appropriate simulation tools to verify their effectiveness.

A suitable tool for performing short-circuit studies in microgrids and modeling contribution from distributed generation, particularly from inverter-interfaced generation, is currently unavailable. Even though software solutions for modeling transient environments-such as MATLAB ${ }^{\circledR}$ Simscape [14], PSIM [15], PLECS ${ }^{\circledR}$ [16] for power electronics and PSCAD [17], EMTP-RV ${ }^{\circledR}$ [18], ATP [19] for power systems-do exist, their high computational costs and data requirements make them unsuitable for distribution network modeling, where the total number of nodes often exceed the $10^{4}$ range.

Modeling inverter-interfaced generation in a conventional phasor-based short-circuit analysis presents difficulties on account of the current-limiting behavior of inverters which introduces nonlinear behavior into the problem. The addition of outer iteration-loops to the short-circuit solver for setting inverter current setpoints becomes necessary [20-25]. This paper discusses how an optimization-based formulation can be employed to perform short-circuit analysis. Such an approach allows for the potential to express protection coordination constraints directly within a design or operation problem (including line switching or generator commitment). These constraints ensure that a given switch state is feasible in terms of protective coordination, so protective devices can isolate a fault without causing an unacceptable number of loads to lose power.

The feasibilities of switch states in smaller microgrids, or in larger microgrids where the number of switch-configurations is limited by operational restrictions, can be determined automatically. An automated approach can be executed sequentially by applying an optimal protection coordination (OPC) problem to each state [26-30]. As relay settings are explicitly calculated, the infeasible states for protection, which otherwise might not be detected by constraints on fault current, can be caught. These approaches are based on performing an offline fault study in advance, where the results provide an input to the OPC [31]. Both the fault study and the OPC must be performed for each switchconfiguration individually.

Performing a fault study to enumerate allowable states becomes infeasible in larger microgrids because the number of possible configurations grows exponentially with the number of switches. In these cases, eliminating the need for protection settings or using novel protection technologies with settings simple enough so that coordination becomes unnecessary are potential solutions to the problem becoming intractable [32,33]. However, for traditional protection, a scalable approach is required. Directly encoding protection feasibility constraints into an optimal operation or planning problem is such an approach. This has been demonstrated for certain use cases, for example, transmission generation capacity expansion [34-37] and fault current limiter placement [38-40]. In these studies, the short-circuit current constraints are encoded using $Z_{b u s}$, the inverse of the admittance matrix. This approach avoids the need to add additional variables for the fault currents. However, it is only valid for conventional synchronous generation where the generator subtransient reactances can be included in $Z_{b u s}$ and $Z_{b u s}$ is relatively constant. This precludes its use in problem formulations that include line switching.

Subsequent work has adapted this $Z_{b u s}$ approach to address the transmission network expansion planning problem that can cause significant changes in $Z_{b u s}$. One approach includes the application of meta-optimization algorithms, such as genetic algorithms [40]. Another applies Bender's decompositions across expansion scenarios [41]. A third creates linear approximations of the nonconvex constraints [42]. Last, the nonlinear constraints are relaxed in order to convexify the problem [43]. All these approaches focus on modeling positive-sequence representations of networks and do not address the additional complexities of modeling unbalanced multi-phase systems that are typical of microgrids. The optimization-based framework presented in this paper was designed to rely on this discussed alternative approach of directly encoding protective feasibility within the microgrid operational problem by constraining fault current to fall within coordination limits. 
However, in this work, the voltages and currents on the system during faults are explicitly included in the formed optimization problem.

Recent work in modeling the short-circuit behavior of grid-connected inverters increasingly focuses on making use of experimental results. Given the current lack of standardization in current-limiting behavior of inverters, this is a necessary step to create accurate models. One such study focuses on validating single- and three-phase dynamic inverter models for load changes against experimental results of commercial grid-forming inverters operating in a stationary reference frame [44]. This study, however, does not take into consideration the current-limiting behavior [45]. Adapting such a model to study short-circuit behavior requires the addition of current-limiting logic, such as described in [23].

Detailed testing of both grid-following and grid-forming inverters under short-circuit conditions has recently been performed in order to provide insight into modeling inverter behavior during faults, including the selection of such logic $[46,47]$. These test results were augmented with field data collected during fault events for grid-following inverters deployed on distribution networks [48]. This has resulted in the availability of dynamic inverter models, which were validated against experimental testing [49,50].

Another development is short-circuit formulations for the fault contributions of gridconnected inverters based on both positive-sequence [51-53], symmetrical component [54], unbalanced three-phase [55], and hybrid [56] representations. These formulations are an important step towards practical short-circuit modeling of grid-connected inverters, but fall short of a unified framework that can address both grid-following and grid forming inverters in a full, unbalanced formulation.

In the case of the presented framework, an optimization-based formulation provides value for inverter-interfaced microgrids by solving for the fault current contribution of inverters running under power factor control mode. The inverter model, which uses a stationary reference frame, is based on [57]. The real and imaginary current contributions become decision variables, whose relative magnitudes are determined by the output bus voltage subjected to current limit constraints.

The implementation of the introduced optimization-based framework for shortcircuit analysis is provided in PowerModelsProtection.jl (https:/ / github.com/lanl-ansi/ PowerModelsProtection.jl accessed on 9 April 2021) (PMsP). PMsP is an extension to PowerModels.jl (https:/ / github.com/lanl-ansi/PowerModels.jl accessed on 9 April 2021) [58] and an addition to the family of free and open-source packages under its umbrella. PowerModels.jl provides a platform to solve and evaluate steady-state power network optimization problems-it decouples problem specifications from the underlying problem formulations, allowing for convex relations to be easily applied. The developed suite of packages include PowerModelsDistribution.jl (https://github.com/lanl-ansi/ PowerModelsDistribution.jl accessed on 9 April 2021) [59], PowerModelsRestoration.jl (https:/ / github.com/lanl-ansi/PowerModelsRestoration.jl accessed on 9 April 2021) [60], PowerModelsGMD.jl. (https:/ / github.com/lanl-ansi/PowerModelsGMD.jl accessed on 9 April 2021) [61]. Together they allow for solving a variety of power systems optimization problems, such as power flow, optimal power flow, minimum load shed, and optimal transmission switching.

PMsP was developed in Julia [62], a high-level, just-in-time compiled programming language designed for scientific computing. Its current main application is for microgrids and distribution networks with distributed energy resources, including both synchronous and inverter-interfaced generation. PMsP is able to model balanced networks by using a positive sequence formulation, as well as unbalanced networks by using a three-wire Kron-reduced representation. Only three-phase faults are supported for balanced networks while for unbalanced networks, arbitrary fault configurations are supported via specifying them with an OpenDSS-style admittance matrix [63]. Both balanced and unbalanced formulations were validated against existing software. In the unbalanced formulation protective 
relaying that requires neutral current measurements can be supported by applying a linear transformation to represent the neutral current.

The key contributions of this paper include the following: (1) formulated short-circuit current flow on unbalanced distribution networks or islanded microgrids in terms of an optimization problem, (2) developed optimization constraints for the short-circuit contribution of inverter-interfaced generation or storage, and (3) formulated constraints for protection coordination applicable to networked microgrids. These developments enable the implementation of planning and operational formulations for microgrids with inverter-interfaced generation, which allows for protection coordination to be maintained, thereby facilitating the reliable operation of large-scale networked microgrids.

The remainder of this paper is organized as follows: Section 2 describes the optimization problem formulation for performing short-circuit studies with inverter-interfaced generation. Section 3 describes how the short-circuit constraints can be embedded into an operation or design problem to ensure protective device coordination. Sections 4 and 5 describe how the short-circuit constraints are applied to the IEEE 123-bus system to perform short-circuit analysis with grid-forming inverters in islanded mode. Finally, Section 6 summarizes the conclusions of this paper about the suitability of optimization-based frameworks for short-circuit analysis.

\section{Short-Circuit Calculations}

A formulation was developed to model the protection scheme of a networked microgrid and add constraints that disallow operating points that do not meet the requirements of an adequately protected system. This formulation for calculating a short-circuit flow (i.e., current triggered by the fault) and the constraints used to determine whether, given the chosen operating point, the protection scheme can isolate the fault, is described in the subsections below. The full list of notation specific to protection scheme modeling is provided in Appendices B and C.

\subsection{Short-Circuit Flow Constraints}

This subsection describes the fault-induced current flows in the protection scheme formulation. Given a set of faults $\mathcal{F}$, the formulation calculates a fault current and voltage for each $f \in \mathcal{F}$ fault. The fault current is used to determine whether the scheme responds correctly and restricts the networked microgrid to operating points that are protected during each fault.

During a fault, synchronous generators are one of the largest sources of injected currents. The current that a certain generator supplies is a function of the pre-fault voltage and the unit's transient reactance. The following constraint defines this:

for $\forall f \in \mathcal{F}, \forall g \in \mathcal{G}_{i}$ :

$$
V_{g f}^{\Phi}=V_{g}^{\Phi}+x_{g} I_{g f}^{\Phi},
$$

where $V_{g}^{\Phi}$ is the internal (pre-fault) voltage and $x_{g}$ is the transient reactance of $g$ generator. This constraint defines the terminal voltage of $g$ as equal to the internal voltage minus the voltage drop created by the injection of the unit and its transient reactance. In the following, this $I_{g f}^{\Phi}$ current is constrained by grid following and grid-forming inverter limits.

Each $f \in \mathcal{F}$ fault is defined by the bus where it occurs, $i_{f}$, and by its admittance matrix, $G_{f}$. The elements of $G_{f}$ depend on the fault type: phase-to-ground or phase-to-phase. The general constraint for calculating fault current at bus $i_{f}$ :

for $\forall f \in \mathcal{F}$ :

$$
I_{s i_{f} f}^{\phi}=G_{f} V_{i_{f}}^{\phi}
$$


Next, the specifics of $G_{f}$ for each fault type are discussed. For a single line-to-ground fault, $G_{f}$ is defined as:

$$
\boldsymbol{G}_{f}=\left[\begin{array}{ccc}
\mathbf{g}_{f} & 0 & 0 \\
0 & 0 & 0 \\
0 & 0 & 0
\end{array}\right] .
$$

where $\mathbf{g}_{f}$ is the admittance of the fault between the phase and ground.

For a line-to-line fault, $G_{f}$ is defined as:

$$
\boldsymbol{G}_{f}=\left[\begin{array}{ccc}
\mathbf{g}_{f} & -\mathbf{g}_{f} & 0 \\
-\mathbf{g}_{f} & \mathbf{g}_{f} & 0 \\
0 & 0 & 0
\end{array}\right],
$$

where $\mathbf{g}_{f}$ is the admittance of the fault between the phases.

For a three-phase ungrounded fault, illustrated in Figure $1, G_{f}$ is based on the delta connection, that is, defined as:

$$
G_{f}=3\left[\begin{array}{ccc}
2 \mathbf{g}_{f} & -\mathbf{g}_{f} & -\mathbf{g}_{f} \\
-\mathbf{g}_{f} & 2 \mathbf{g}_{f} & -\mathbf{g}_{f} \\
-\mathbf{g}_{f} & -\mathbf{g}_{f} & 2 \mathbf{g}_{f}
\end{array}\right],
$$

where $\mathbf{g}_{f}$ is the admittance of the fault between the phases.
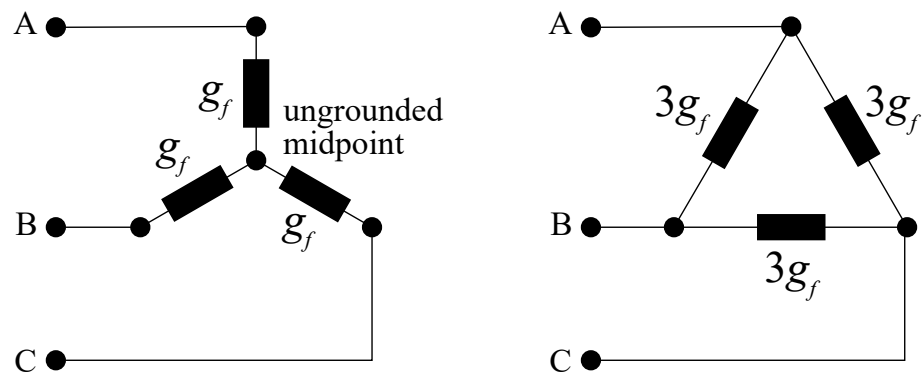

Figure 1. Star-mesh transformation for a three-phase ungrounded fault.

For a line-line-ground fault, illustrated in Figure 2, a star-mesh transformation is performed in order to calculate the values of elements of $G_{f}$, based on the resistances between the phases $\left(\mathbf{g}_{p}\right)$ and the ground $\left(\mathbf{g}_{g}\right)$, where

$$
\begin{gathered}
\mathbf{g}_{\text {total }}=2 \mathbf{g}_{p}+\mathbf{g}_{g} \\
\mathbf{g}_{p p}=\frac{\mathbf{g}_{p}^{2}}{\mathbf{g}_{\text {total }}} \\
\mathbf{g}_{p g}=\frac{\mathbf{g}_{p} \mathbf{g}_{g}}{\mathbf{g}_{\text {total }}} .
\end{gathered}
$$

The $G_{f}$ matrix for a line-line-ground fault is then defined as:

$$
\boldsymbol{G}_{f}=\left[\begin{array}{ccc}
\mathbf{g}_{p p} \mathbf{g}_{p g} & -\mathbf{g}_{p p} & 0 \\
-\mathbf{g}_{p p} & \mathbf{g}_{p p} \mathbf{g}_{p g} & 0 \\
0 & 0 & 0
\end{array}\right] .
$$



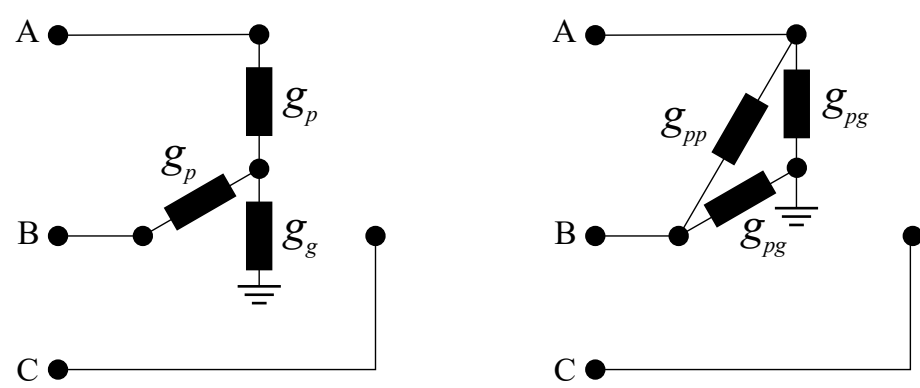

Figure 2. Star-mesh transformation for a line-line-ground fault.

The same star-mesh transformation is used to calculate $G_{f}$ for a three-phase grounded fault, illustrated in Figure 3:

$$
\begin{gathered}
\mathbf{g}_{\text {total }}=3 \mathbf{g}_{p}+\mathbf{g}_{g} \\
\mathbf{g}_{p p}=\frac{\mathbf{g}_{p}^{2}}{\mathbf{g}_{\text {total }}} \\
\mathbf{g}_{p g}=\frac{\mathbf{g}_{p} \mathbf{g}_{g}}{\mathbf{g}_{\text {total }}} .
\end{gathered}
$$

The $G_{f}$ matrix for a three-phase to ground fault is then defined as:

$$
\boldsymbol{G}_{f}=\left[\begin{array}{ccc}
2 \mathbf{g}_{p p} \mathbf{g}_{p g} & -\mathbf{g}_{p p} & -\mathbf{g}_{p p} \\
-\mathbf{g}_{p p} & 2 \mathbf{g}_{p p} \mathbf{g}_{p g} & -\mathbf{g}_{p p} \\
-\mathbf{g}_{p p} & -\mathbf{g}_{p p} & 2 \mathbf{g}_{p p} \mathbf{g}_{p g}
\end{array}\right]
$$
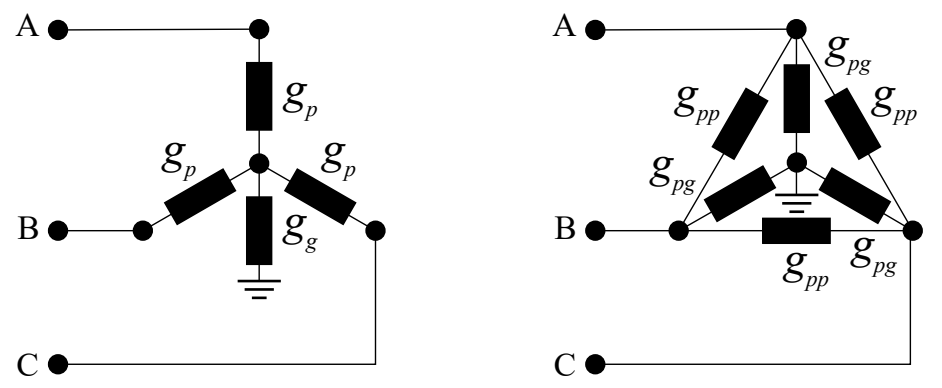

Figure 3. Star-mesh transformation for a three-phase to ground fault.

Kirchhoff's current law is followed to ensure that the current injections at each bus are balanced. For non-faulted buses, the balancing constraint is defined as:

for $\forall f \in \mathcal{F}, \forall g \in \mathcal{G}_{i}, \forall i \in \mathcal{N} \backslash i_{f}, \forall \phi \in \Phi_{i}:$

$$
\sum_{(i, j, k) \in \mathcal{E}_{i}^{+}} I_{i j k f}^{\phi}-\sum_{(j, i, k) \in \mathcal{E}_{i}^{-}} I_{i j k f}^{\phi}=\sum_{g \in \mathrm{G}(i)} I_{g f}^{\phi}-I_{i_{f} f^{\prime}}^{\phi}
$$

where the terms on the left side represent the currents of lines and transformers connected to the bus, while the terms on the right side represent the current injections into bus from equipment located at the bus, generators-including any equipment that can provide power (e.g., diesel gensets, photovoltaic (PV) generation or battery energy storage)—and loads.

For faulted buses, the balancing constraint contains $I_{s i_{f} f}^{\phi}$ fault current as defined in constraint (2):

for $\forall f \in \mathcal{F}, \forall g \in \mathcal{G}_{i}, \forall i \in \mathcal{N} \backslash i_{f}, \forall \phi \in \Phi_{i}$ :

$$
\sum_{(i, j, k) \in \mathcal{E}_{i_{f}^{+}}^{+}} I_{i j k f}^{\phi}-\sum_{(j, i, k) \in \mathcal{E}_{i_{f}^{-}}^{-}} I_{i j k f}^{\phi}=\sum_{g \in \mathrm{G}\left(i_{f}\right)} I_{g f}^{\phi}-I_{s i_{f} f}^{\phi}-I_{i_{f} f}^{\phi} .
$$


To complete this subsection, constraints (1), (2), (14), and (15) are added to the formulation to calculate fault currents for protection scheme modeling.

\subsection{Short-Circuit Flow Formulation}

Constraints (14) and (15)-introduced in Section 2.1-are applied to the currentvoltage formulation of PowerModelsDistribution.j1 [59]. The optimization of PMsP is formulated to calculate the voltages for each bus and the three-phase currents for each system component. The formulation used by PMsP for calculating fault currents is presented below. It should be noted that the formulation is based in rectangular coordinates. In order to simplify presentation, the constraints are not explicitly separated into real and imaginary parts. This formulation is expressed a constraint satisfaction problem because all devices are operating under local control. There is no objective function, with the optimizer searching only for a feasible solution that satisfies all of the constraints. The current balancing constraint provides the main source of coupling between multiple nodes in the problem. These constraints can be applied as an additional set of constraints to an optimization problem such as an optimal power flow or optimal switching.

Subject to:

for $\forall g \in \mathcal{G}, \forall \phi \in \Phi_{i}:$

$$
\begin{gathered}
V_{g_{\text {ref }}}^{\phi}=V_{g_{0}}^{\phi} \\
V_{g f}^{\phi}=V_{g_{0} f}^{\phi}-Z_{g}^{\phi} I_{g f}^{\phi}
\end{gathered}
$$

for $\forall g \in \mathcal{G}, \forall f \in \mathcal{F}, \forall i \in \mathcal{N} \backslash i_{f}, \forall \phi \in \Phi_{i}:$

$$
\begin{gathered}
\sum_{(i, j, k) \in \mathcal{E}_{i}^{+}} I_{i j k f}^{\phi}-\sum_{(j, i, k) \in \mathcal{E}_{i}^{-}} I_{i j k f}^{\phi}=\sum_{g \in \mathcal{G}_{i}} I_{g f}^{\phi}-I_{i_{f} f}^{\phi} \\
\sum_{(i, j, k) \in \mathcal{E}_{i_{f}}^{+}} I_{i j k f}^{\phi}-\sum_{(j, i, k) \in \mathcal{E}_{i_{f}}^{-}} I_{i j k f}^{\phi}=\sum_{g \in \mathcal{G}_{i_{f}}} I_{g f}^{\phi}-I_{s i_{f} f}^{\phi}-I_{i_{f} f}^{\phi}
\end{gathered}
$$

for $\forall g \in \mathcal{G}, \forall i \in \mathcal{N}, \forall \phi \in \Phi_{i}, \forall(i, j, k) \in \mathcal{E}_{i}:$

$$
I_{i f}^{\phi}=I_{i j k f}^{\phi}+\left(g_{i j k f}^{\phi}+j b_{i j k f}^{\phi}\right) V_{i f}^{\phi}
$$

for $\forall g \in \mathcal{G}, \forall i \in \mathcal{N}, \forall \phi \in \Phi_{i}, \forall(j, i, k) \in \mathcal{E}_{i}:$

$$
\begin{aligned}
& I_{j f}^{\phi}=I_{j i k f}^{\phi}+\left(g_{j i k f}^{\phi}+j b_{j i k f}^{\phi}\right) V_{j f}^{\phi} \\
& V_{j f}^{\phi}=V_{i f}^{\phi}+\left(r_{i j k f}^{\phi}+j x_{i j k f}^{\phi}\right) I_{i j k f}^{\phi}
\end{aligned}
$$

for $\forall f \in \mathcal{F}, \forall i \in \mathcal{N}:$

$$
I_{s i_{f} f}^{\Phi}=G_{i f} V_{i f}^{\Phi}
$$

for $\forall i, j \in \mathcal{N}, \forall(i, j, k) \in \chi:$

$$
\begin{aligned}
& W_{i j k} V_{i f}=\eta_{i j k} V_{j f} \\
& W_{i j k}{ }^{\prime} I_{j f}=\eta_{i j k} I_{i f} .
\end{aligned}
$$

Constraint (16) sets the magnitude and angle of the generator connected to the reference bus. As with other fault formulations, a generator is defined as a voltage source behind an impedance, which is shown in constraint (17), where the voltage $V_{g_{0}}$ is the prefault voltage of the generator. In the case of inverter-interfaced generation, constraint (16) is replaced with an additional set of constraints governing $I_{g f}^{\phi}$. These constraints are de- 
pendent on whether the inverter is operating in grid-following or grid-forming mode (presented in Sections 2.3 and 2.4). Constraints (18) and (19) are the current balancing constraints discussed in Section 2.1. Constraint (20) and (21) define the current injected into a bus from a line, while constraint (22) defines the voltage drop across the line. Figure 4 illustrates the interactions between two adjacent buses in a three-phase model (sub-figures are corresponding to phase voltages). Constraint (23) defines the fault current. The last set of constraints, constraints (24) and (25), define the voltages and currents for transformers, where $\eta_{i j k}$ represents the scaling of voltages and currents due to the winding ratios and the matrix $W_{i j k}$ defines the connections of transformers.

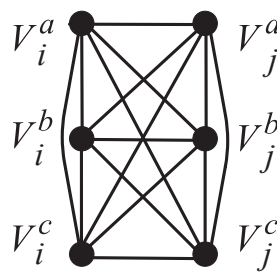

(a)

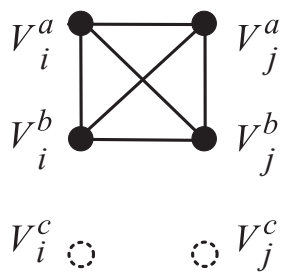

(b)

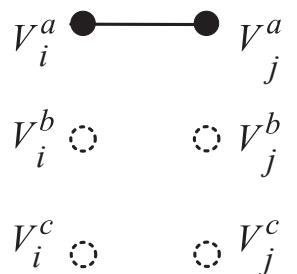

(c)
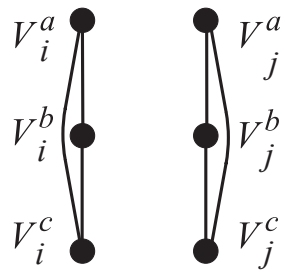

(d)

Figure 4. Sub-figures $(\mathbf{a}-\mathbf{c})$ present two adjacent three-, two-, and single-phase buses that are connected via a distribution line. Sub-figure (d) presents two adjacent three-phase buses that are connected via an ideal transformer [64].

Although the focus of this paper is on fault current contributions from inverterinterfaced generation, it is important to validate the developed short-circuit flow formulation (defined by constraints (16)-(25)) as PMsP relies on it. Results are provided in Table 1. The produced fault currents were compared to results from OpenDSS [63] and provided by the IEEE Power and Energy Society [65]. The IEEE 123-Node System (shown in Figure 5) was used, with faults placed at Buses 13, 67, and 113. The results of the formulation were within $5 \%$ of both the results produced by OpenDSS and the results provided by IEEE for this Short-Circuit Test Case.

Table 1. Comparison of results for short-circuit flow formulation.

\begin{tabular}{ccccccc}
\hline Fault Node & Fault Phasing & $\begin{array}{c}\text { IEEE PES } \\
\text { Fault Current (A) }\end{array}$ & $\begin{array}{c}\text { OpenDSS } \\
\text { Fault Current (A) }\end{array}$ & $\begin{array}{c}\text { IEEE-DSS } \\
\text { Difference (\%) }\end{array}$ & $\begin{array}{c}\text { PMsP } \\
\text { Fault Current (A) }\end{array}$ & $\begin{array}{c}\text { IEEE-PMsP } \\
\text { Difference (\%) }\end{array}$ \\
\hline 13 & LG & 4400.1 & 4422.0 & +0.50 & 4444.9 & +1.02 \\
13 & LL & 4886.6 & 4736.0 & -3.08 & 4954.2 & +1.38 \\
13 & 3P & 5435.3 & 5305.0 & -2.40 & 5517.3 & +1.51 \\
67 & LG & 2339.5 & 2392.0 & +2.24 & 2362.2 & +0.97 \\
67 & LL & 2890.0 & 2879.0 & -0.38 & 2933.1 & +1.49 \\
67 & 3P & 3236.6 & 3251.0 & +0.44 & 3285.6 & +1.51 \\
113 & LG & 1382.6 & 1390.0 & +0.54 & 1392.2 & +0.69 \\
\hline
\end{tabular}




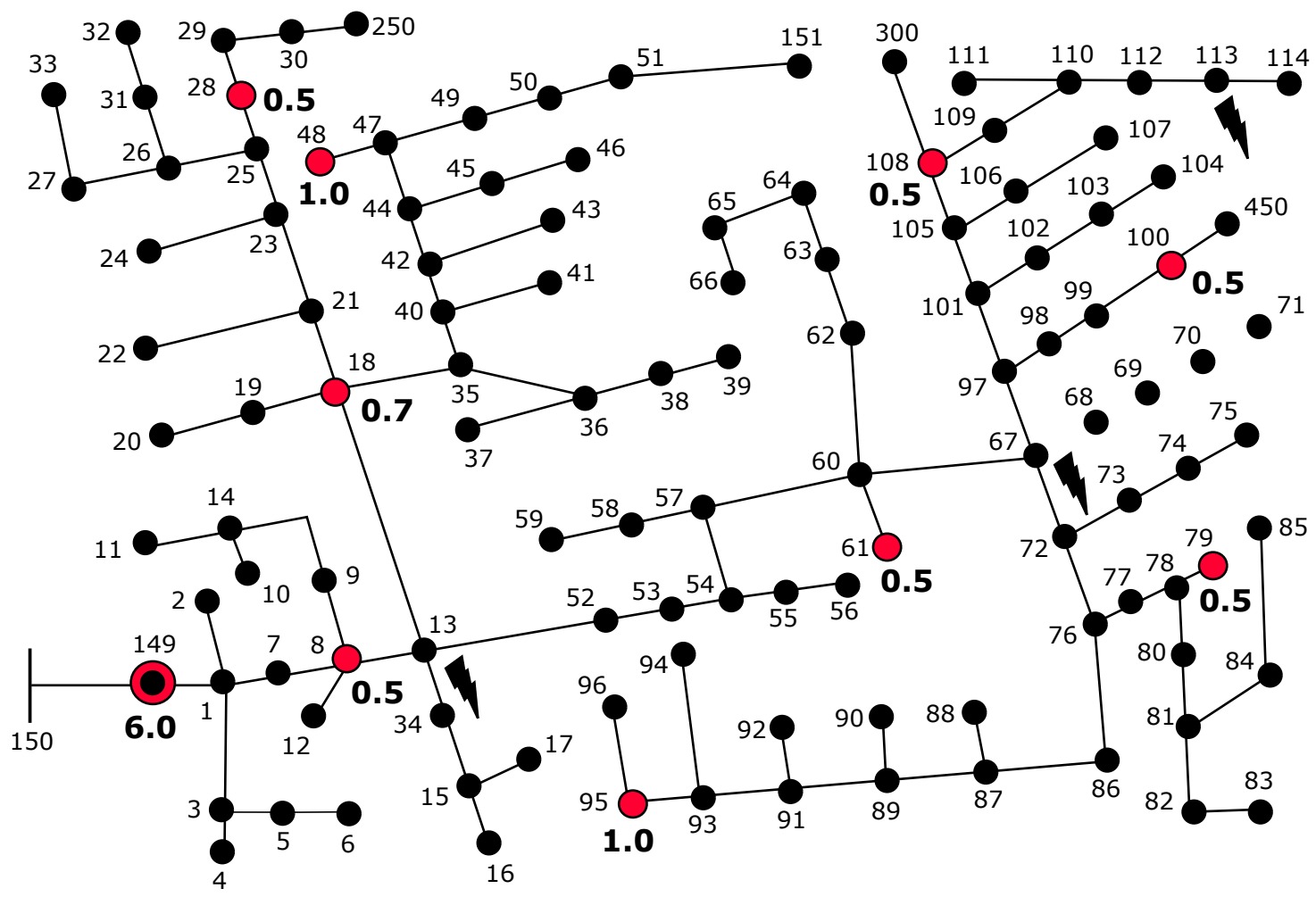

Figure 5. The IEEE 123-Node System [66].

\subsection{Grid-Following Inverter Constraints}

The following Subsections describe the additional constraints that inverters impose on the short-circuit flow constraints introduced in Section 2.1. For the purposes of short-circuit calculations, grid-following and grid-forming inverters are distinguished. Typically, gridfollowing inverters (Section 2.3) operate as distributed energy resources (e.g., residential or community-scale solar installations). Grid-forming inverters (Section 2.4), on the other hand, are intended for standalone operation (e.g., used in off-grid solar systems) or are designed specifically for microgrids that are capable of islanded operation. Grid-following inverters behave as a constant-power source under normal operation, while grid-forming inverters behave as a constant-voltage source [67-69].

Grid-following inverters can have a non-negligible contribution to fault currents in networked microgrids connected to the bulk power system through a high impedance or in islanded microgrids [70]. Design and operating principles of a grid-following inverter are described in detail in [71]. While they nominally operate as a constant-power source, during fault conditions the output terminal voltage may drop to sufficiently low limits so that the inverter is unable to meet its power setpoint without exceeding its current limits. These current limits are determined by the thermal rating of the power semiconductor switches in the inverter. Due to the low thermal time-constant of power semiconductors compared to rotating generation, the inverter will switch to current-limiting mode within cycles of a fault appearing. The introduced formulation assumes that the inverter supplies only real power and positive-sequence current. It is also assumed that the inverter will switch between normal and current limiting operation depending on grid conditions.

First the complex term $\alpha$ is defined, which is needed to calculate the sequence components:

$$
\begin{array}{rlrl}
\alpha & =e^{\mathrm{j} 2 \pi / 3} & \alpha^{2}=e^{-\mathrm{j} 2 \pi / 3} \\
\alpha_{r}=\Re(\alpha)=-\frac{1}{2} & \alpha_{i}=\Im(\alpha)=\frac{\sqrt{3}}{2} \\
\alpha_{r}^{2}=\Re(\alpha)^{2}=-\frac{1}{2} & \alpha_{i}^{2}=\Im(\alpha)=-\frac{\sqrt{3}}{2},
\end{array}
$$


where the notation $\Re$ and $\Im$ is used to denote the real and imaginary parts of a complex number. The term $\alpha$ is commonly used within the transformation matrices $A$ and $A_{i}$ but for this work the scalar representation is used instead.

Once the transients of the fault subdue, the inverter injects positive-sequence current resulting in three sets of constraints applied to the sequence components of gridfollowing inverters:

for $\forall f \in \mathcal{F}, \forall g \in \mathcal{G}_{p q i}$ :

$$
\begin{gathered}
\sum_{\phi \in \Phi} I_{g f}^{\phi}=0 \\
0=I_{g f}^{a}+\alpha^{2} I_{g f}^{b}+\alpha I_{g f}^{c} \\
3 I_{g f}^{+}=I_{g f}^{a}+\alpha I_{g f}^{b}+\alpha^{2} I_{g f}^{c},
\end{gathered}
$$

where (26) is the zero sequence constraint, (27) is the negative sequence constraint, and (28) is the positive sequence constraint. In constraints (27) and (28), $\alpha$ is used to transform from the phase reference frame to the sequence component frame.

During a fault, the only injected current from the grid-following inverter is the positive sequence component, so the constraints are based on the positive sequence voltage and current. This work assumes that the inverter is constrained to operate at unity power factor, which results in the following constraints:

Subject to:

for $\forall f \in \mathcal{F}, \forall g \in \mathcal{G}_{p q i}, \forall \phi \in \Phi$ :

$$
\begin{gathered}
\sum_{\phi \in \Phi} p_{g f}^{\phi}=3 \Re\left(V_{g f}^{+}\left(I_{g f}^{+}\right)^{*}\right) \\
\sum_{\phi \in \Phi} q_{g f}^{\phi}=0=3 \Im\left(V_{g f}^{+}\left(I_{g f}^{+}\right)^{*}\right) \\
0 \leqslant \sum_{\phi \in \Phi} p_{g f}^{\phi} \leqslant \bar{p}_{g} \\
\left|I_{g f}^{+}\right| \leqslant \bar{I}_{g_{f}} \\
I_{g f}^{+}=\bar{I}_{g f} \\
I_{g f}^{+}=\kappa_{g} \bar{I}_{g f} \\
\left(I_{g f}^{+}-\bar{I}_{g f} z_{g f}\right) \geqslant 0 \\
p_{g f} \geqslant\left(1-z_{g f}\right) \hat{p} .
\end{gathered}
$$

Note that in constraints (29) and (30) the zero limit is not included. Constraint (31) defines that the inverter real power is subjected to the inverter maximum power limit. Constraint (32) restricts the magnitude of the actual current to be less than the rated current, while constraint (33) restricts the magnitude of the current under current-limiting conditions to be equal to the rated current. Constraint (34), where $\kappa_{g} \in[0,1]$, restricts the phasor quantities for the actual current to be between the origin and the injected current under current-limiting mode. Constraint (35), where $z_{g_{f}} \in[0,1]$ and the inverter is in current operation when $z_{g_{f}}=1$, restricts the actual current of the inverter to the maximum value if $z_{g_{f}}=1$. Last, constraint (36) restricts the injected power to be equal to the scheduled power when the inverter is not in current-limiting operation.

Constraints (26)-(36) are added to the formulation to aid in calculating fault currents. The relationships between the inverter terminal voltage, current and power resulting from these constraints are illustrated graphically in Figure 6. 


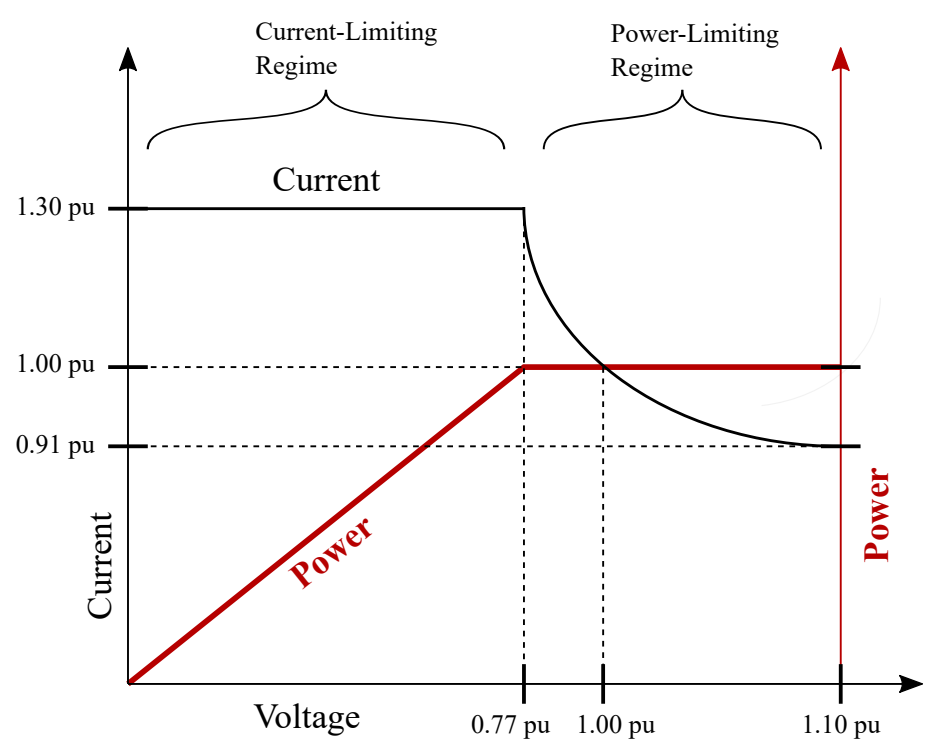

Figure 6. Graphical illustration of constraints for a grid-following inverter.

\subsection{Grid-Forming Inverter Constraints}

As mentioned before, grid-forming inverters nominally operate as a constant-voltage source [70]. The implication here is that while grid-following inverters are constrained to inject real power and positive-sequence current (see Section 2.3), grid-forming inverters do not have these constraints. Additionally, these inverters toggle between normal and current-limiting operation on a per-phase basis. The grid-forming inverter constraints are the following:

Subject to:

for $\forall f \in \mathcal{F}, \forall g \in \mathcal{G}_{v i}, \forall \phi \in \Phi:$

$$
\begin{gathered}
I_{g f}^{\phi} \leqslant \bar{I}_{g f} \\
\left(I_{g f}^{\phi}-\bar{I}_{g f}\right) z_{g f}^{\phi} \geqslant 0 \\
\left|\sum_{\phi \in \Phi} p_{g f}^{\phi}\right| \leqslant\left|\Re\left(V_{g f}\left(I_{g f}\right)^{*}\right)\right| \\
\left|I_{g f}^{\phi}\right| \leqslant \bar{I}_{g f} \\
V_{g f}^{\Phi}=V_{g f}^{\Phi s e t}-Z_{g f}^{\text {quirtual }} I_{g f}^{\Phi},
\end{gathered}
$$

where $Z_{g f}^{\Phi}$ is a set of decision variables that are based on the current limiting indicator variable $z_{g f}^{\Phi}$ and allow the inverter's terminal voltage to adjust to match the system. $Z_{g f}^{\Phi}$ is defined as:

$$
\begin{gathered}
Z_{g f}^{\Phi \text { virtual }}=r_{g f}^{\Phi}+j x_{g f}^{\Phi} \\
0 \leqslant r_{g}^{\Phi} \leqslant \hat{r}_{g} \quad 0 \leqslant x_{g}^{\Phi} \leqslant \hat{x}_{g},
\end{gathered}
$$

where the parameters $\hat{r}_{g}$ and $\hat{x}_{g}$ set the upper limit of the virtual resistance and impedance.

$$
\begin{gathered}
V_{g f}^{\Phi s e t} \leqslant V_{g f}^{\Phi r e f}+\boldsymbol{M}_{g} z_{g f}^{\phi} \\
V_{g f}^{\Phi s e t} \geqslant V_{g f}^{\Phi r e f}-\boldsymbol{M}_{g} z_{g f}^{\phi} .
\end{gathered}
$$

Constraint (37) is the per-phase current limiting constraint, and constraint (38) is a similar per-phase lower limit constraint for current-limiting mode of grid-forming inverters. Constraint (39) defines that the total power across all phases must not exceed the power 
supplied by the source at the dc-bus (regardless if it is PV solar, battery, diesel generator, etc.), while constraint (40) restricts the magnitude of the actual current to be less than the rated current. Constraint (41) is a voltage drop constraint on the voltage setpoints that account for virtual impedances used for power sharing. Constraints (43) and (44) allow for the reference voltage $V_{g f}^{\Phi r e f}$ to adjust when the inverter reaches the current limits, while also ensuring that the set point of the inverter is equal to the reference voltage when not in current limiting mode.

Constraints (37)-(44) are added to the formulation to aid in calculating fault currents. The relationships between the inverter terminal voltage, current and the Thevenin admittance of network resulting from these constraints are illustrated graphically in Figure 7.

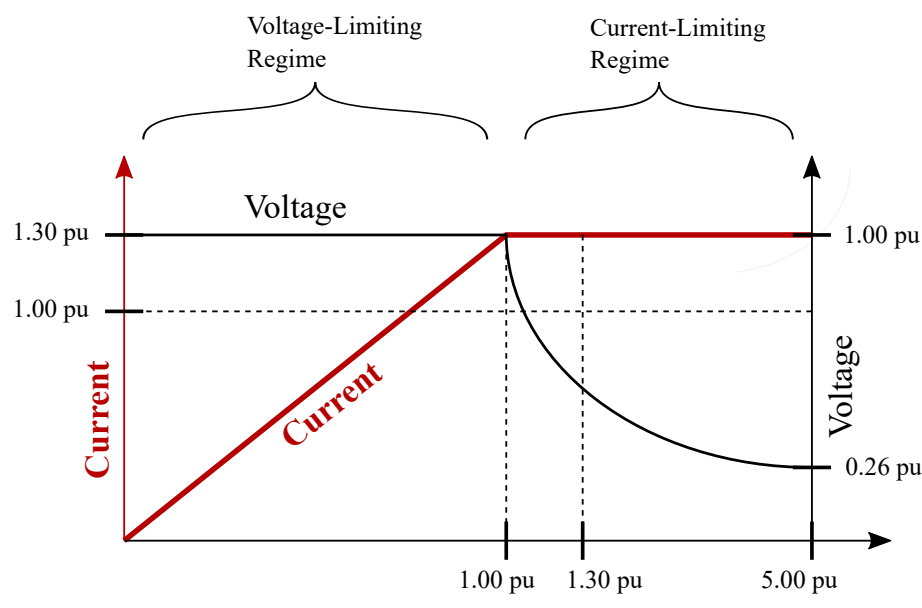

Thevenin Admittance

Figure 7. Graphical illustration of constraints for a grid-forming inverter.

\section{Protection Coordination Constraints}

The motivation behind performing short-circuit analysis on a microgrid is to evaluate whether protection coordination is achieved. This section considers the concept of developing a set of constraints that determine when protection operates, and to relate them to power supplied by the microgrid post-fault, allowing for the quality of protection coordination to be quantified in terms of the amount of load lost. The constraints presented here can currently be evaluated sequentially on the short-circuit results, although a relaxed or approximated version could be directly incorporated into a microgrid operational problem.

\subsection{Perfect Knowledge Protection}

The simplest approach for studying the behavior of microgrid protection is to assume perfect protection operation, as illustrated in Figure 8. In this case, there is a set of $\mathcal{Z}$ protective zones. Each zone $\zeta \in \mathcal{Z}$ is associated with a set of $\mathcal{P}_{\zeta}$ protective devices. Each zone protects $\mathcal{B}_{\zeta}$ nodes, while each protective device $(i, j, k)$ is associated with a branch terminal. For a fault located in the set $\mathcal{B}_{\zeta}$, the branches associated with that set of protective devices become inoperable.

The impact of protective device operation in terms of load lost can then be expressed as a restoration minimum-load shed problem, where branches associated with a tripped state have indicator variable $\delta_{i j k r}=0$ and those not tripped have $\delta_{i j k r}=1$. 


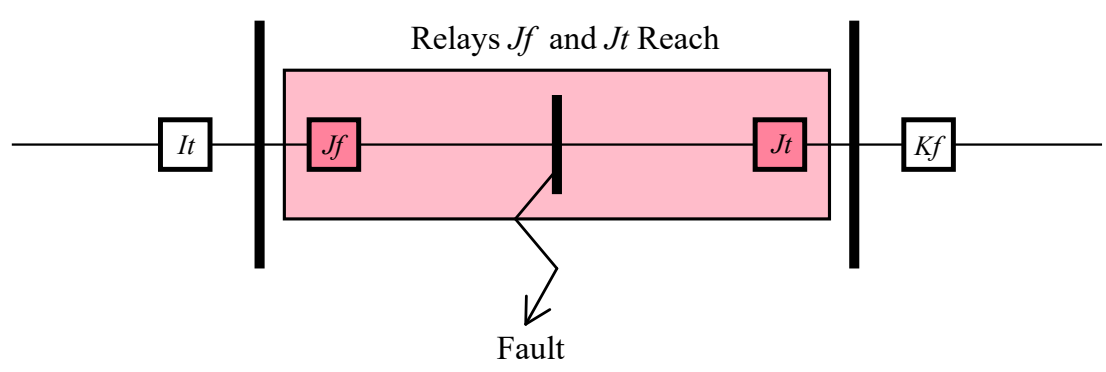

Figure 8. Oneline diagram for an ideal protection scheme.

The implemented three-phase power flow [64] is established based on current (I), voltage $(V)$, admittance $(Y)$, and power $(S)$. These quantities are defined on a per phase basis, $\Phi=\{a, b, c\}$, which is linked through voltage and admittance. The full list of notation specific to three-phase power flow are provided in Appendices A and D.

In an unbalanced three-phase configuration, the power flow on a line $(i, j, k)$ and phase $\phi$ is affected by the voltages on all three phases according to the conductance and susceptance values appearing in the admittance matrix $Y_{i j k}^{\Phi}$. This relationship is expressed as follows:

for $\forall(i, j, k) \in \mathcal{E}$ :

$$
\begin{gathered}
I_{i j k r}^{\Phi}=\delta_{i j k r} Y_{i j k}^{\Phi}\left(V_{i r}^{\Phi}-V_{j r}^{\Phi}\right) \\
\sum_{g \in \mathcal{G}_{i}} \delta_{g r} S_{g r}^{\Phi}-\delta_{i r} S_{i r}^{\Phi}=\sum_{(i, j, k) \in \mathcal{E}_{i}^{+}} S_{i j k r}^{\Phi}-\sum_{(j, i, k) \in \mathcal{E}_{i}^{-}} S_{j i k r}^{\Phi}
\end{gathered}
$$

where the first equation can be expanded to:

$$
\left[\begin{array}{l}
I_{i j k r}^{a} \\
I_{i j k r}^{b} \\
I_{i j k r}^{c}
\end{array}\right]=\delta_{i j k r}\left[\begin{array}{ccc}
\boldsymbol{Y}_{i j k}^{a a} & \boldsymbol{Y}_{i j k}^{a b} & \boldsymbol{Y}_{i j k}^{a c} \\
\boldsymbol{Y}_{i j k}^{a b} & \boldsymbol{Y}_{i j k}^{b b} & \boldsymbol{Y}_{i j k}^{b c} \\
\boldsymbol{Y}_{i j k}^{a c} & \boldsymbol{Y}_{i j k}^{b c} & \boldsymbol{Y}_{i j k}^{c c}
\end{array}\right] \cdot\left[\begin{array}{c}
\left(V_{i r}^{a}-V_{j r}^{a}\right) \\
\left(V_{i r}^{b}-V_{j j}^{b}\right) \\
\left(V_{i r}^{c}-V_{j r}^{c}\right)
\end{array}\right]
$$

The above equations lead to the following representation: for $\forall(i, j, k) \in \mathcal{E}, \forall \phi \in \Phi_{i j k}$ :

$$
\begin{gathered}
I_{i j k r}^{\phi}=\sum_{\hat{\phi} \in \Phi_{i j k}} \delta_{i j k r} Y_{i j k}^{\phi \hat{\phi}}\left(V_{i r}^{\hat{\phi}}-V_{j r}^{\hat{\phi}}\right) \\
S_{i j k r}^{\phi}=V_{i r}^{\phi} \sum_{\hat{\phi} \in \Phi_{i j k}} \delta_{i j k r}\left(Y_{i j k}^{\phi \hat{\phi}}\right)^{*}\left(V_{i r}^{\hat{\phi}}-V_{j r}^{\hat{\phi}}\right)^{*},
\end{gathered}
$$

where $\Phi_{i j k}$ denotes the phases supported by line $(i, j, k)$.

In case of perfect knowledge protection, the objective of the problem formulation is to maximize the load supplied, subjected to constraints (45a)-(47b) and bounds on current, voltage and power:

$$
\max \sum_{i \in \mathcal{B}} \sum_{\phi \in \Phi_{i}} \delta_{i r} P_{i r}^{\phi}
$$

\subsection{Pilot Protection}

In the case of pilot protection, it is assumed that protective relays are installed in sets of $\mathcal{P}_{\zeta}$ relays, where each relay is again associated with a branch terminal, as illustrated in Figure 4 , and typically the set cardinality is 2 . For protection, the same quantities are used as that of Dewadasa et al. [5,7], where line-ground faults are detected by operating on the measured positive-sequence impedance between the relay and the fault: 
for $\forall(i, j, k) \in \mathcal{P}, \forall \phi \in \Phi_{i j k}$ :

$$
\tilde{Z}_{i j k f}^{l g+}=\frac{V_{i f}^{\phi}}{I_{i j k f}^{\phi}+k_{i j k f} I_{i j k f}^{0}} .
$$

Similarly, line-line faults are also detected by operating on the measured positivesequence impedance:

for $\forall(i, j, k) \in \mathcal{P}, \forall \phi \in \Phi_{i j k}$ :

$$
\tilde{Z}_{i j k f}^{l l+}=\frac{V_{i f}^{\psi}-V_{i f}^{\phi}}{I_{i j k f}^{\phi}-I_{i j k f}^{\psi}} .
$$

The quantity $k_{i j k f}$ is related to the ratio of measured positive- and negative-sequence line impedance as:

for $\forall(i, j, k) \in \mathcal{P}$ :

$$
k_{i j k f}=1-\frac{\tilde{Z}_{i j k f}^{0}}{\tilde{Z}_{i j k f}^{+}} .
$$

A trip will occur when all relays in the set detect a trip. A directional comparison blocking scheme (DCB), illustrated in Figures 9 and 10, is recommended [12]. However, for the purposes of analysis, pilot relaying is assumed to be ideal and error-free. This is expressed in terms of the restoration branch status as:

for $\forall(i, j, k) \in \mathcal{P}$ :

$\delta_{i j k r}= \begin{cases}0, & \left(\tilde{Z}_{i j k f}^{l g+} \in \mathcal{Z}_{i j k}^{l g, o p}\right) \wedge\left(\Re\left(I_{i j k f}^{-} / V_{i f}^{a}\right) \geqslant 0\right) \wedge\left(\tilde{Z}_{j i k f}^{l g+} \in \mathcal{Z}_{j i k}^{l g, o p}\right) \wedge\left(\Re\left(I_{j i k f}^{-} / V_{j f}^{a}\right) \geqslant 0\right) \\ 0, & \left(\tilde{Z}_{i j k f}^{l l+} \in \mathcal{Z}_{i j k}^{l l, o p}\right) \wedge\left(\Re\left(I_{i j k f}^{-} / V_{i f}^{a}\right) \geqslant 0\right) \wedge\left(\tilde{Z}_{j i k f}^{l l+} \in \mathcal{Z}_{j i k}^{l l, o p}\right) \wedge\left(\Re\left(I_{j i k f}^{-} / V_{j f}^{a}\right) \geqslant 0\right) \\ 1, & \text { otherwise, }\end{cases}$

which serves as a constraint to set $\delta_{i j k}$, given the minimum-load-shed formulation in (45a)-(48).

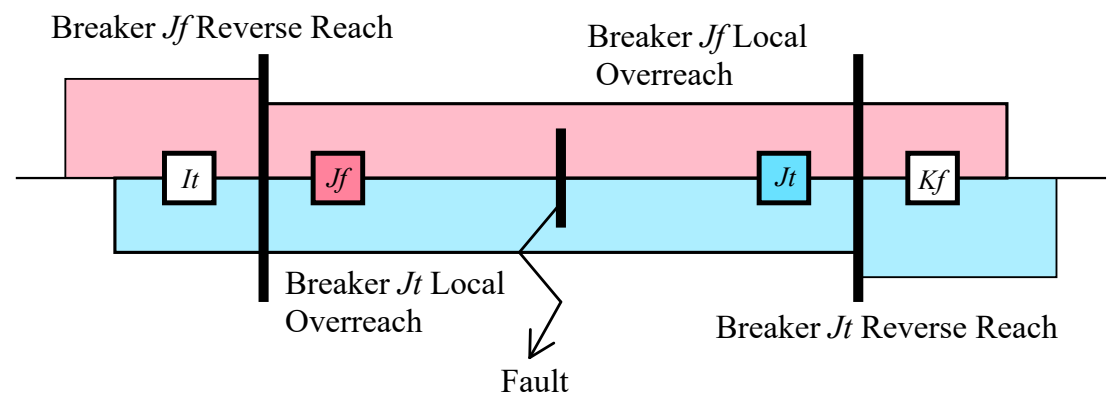

Figure 9. Oneline diagram for a DCB scheme.

Forward Distance

Fault Detector

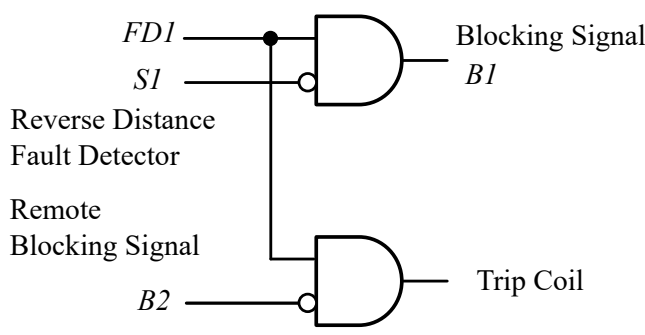

Figure 10. Schematic for one relay in a directional comparison blocking (DCB) scheme. 


\subsection{Time-Overcurrent Protection}

In case of time-overcurrent protection [26,72], which is typically used for recloser-fuse coordination, first the operating time of a protective device is determined:

for $\forall(i, j, k) \in \mathcal{P}$

$$
t_{i j k}^{p}=\min _{\phi \in \Phi} \frac{A \cdot T_{i j k} \cdot \max \left(\Re\left(I_{i j k f}^{\phi}\right), 0\right)}{\left(I_{i j k f}^{\phi} / I_{i j k}^{p}\right)^{B}-1},
$$

where $T_{c j}$ is the time-dial setting and $I_{c j}^{p}$ is the pickup current setting, which are decision variables for the OPC problem (both are subjected to upper and lower bounds). $\max \left(\Re\left(I_{i j k f}^{-} / V_{i f}^{a}\right), 0\right)$ is a directional element that prevents the recloser from operating for upstream faults, even in the presence of distributed generation. This is illustrated for two different values of $I_{i j k}^{p}$ in Figure 11.

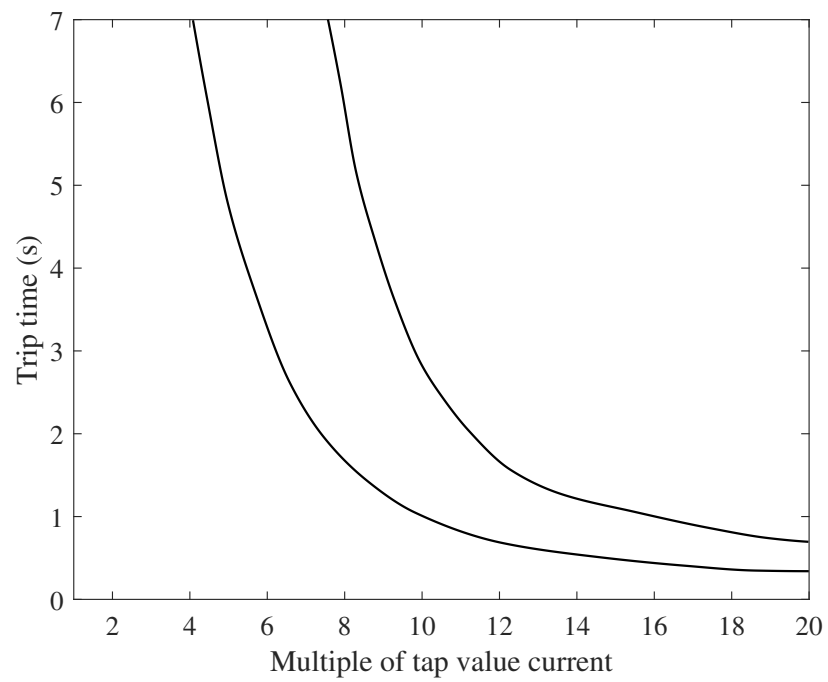

Figure 11. Examples of time-overcurrent relay curves [20].

The operating time of the recloser that trips during a fault is that of the minimum $t_{i j k}^{p}$ of the set of reclosers in the microgrid:

for $\forall(i, j, k) \in \mathcal{P}$ :

$$
\begin{gathered}
\delta_{i j k r} t_{(1)}^{p} \leqslant t_{i j k}^{p}-T_{\mathcal{C}} \\
t_{(1)}^{p}=\sum_{i j k} t_{i j k}^{p}\left(1-\delta_{i j k r}\right) \quad \sum_{i j k \in \mathcal{E}_{p}}\left(1-\delta_{i j k r}\right)=1 .
\end{gathered}
$$

Note that (55) requires that exactly one recloser operate during a fault. Additionally, there is a constraint on maximum operating time:

$$
0 \leqslant t_{(1)}^{p} \leqslant \hat{t}^{p}
$$

It is common practice in optimal protection literature to simplify the problem formulation by not explicitly considering post-fault power flow, but rather to group protective devices into pairs and require that the downstream device in each pair operate before the upstream device is subjected to a coordination time interval [26].

\subsection{Approximating Constraint Formulation}

While implementing both the ideal "perfect knowledge" protection constraints (Section 3.1) and the pilot protection constraints (Section 3.2) in an optimization formulation is a straightforward task, the time-overcurrent protection constraints (Section 3.3) pose a challenge in that (1) they include highly nonconvex constraints, and (2) the range 
for the operation time variables can be very large, which leads to numerical problems. Consequently, although global optimization methods exist, OPC problem formulations typically are restricted to heuristic search methods (e.g., genetic algorithms). To embed time-overcurrent constraints within an operation or design problem, the upper and lower current coordination limits can be employed to place bounds on the allowable fault current. This can be done sequentially for smaller sized systems.

\section{Case Studies}

To demonstrate how the above introduced optimization problem formulations can be employed for short-circuit analysis and protective relaying design of networked microgrids, validation case studies were performed on a handful of grid-connected and islanded configurations.

\subsection{IEEE 123-Node System}

The IEEE 123-Node System [65,66] (shown in Figure 5) was used to test the gridfollowing and grid-forming inverter constraints. The implementation of the system is based on the IEEE 123-Node Short-Circuit Case provided with the OpenDSS source code [63]. This was modified by adding distributed generation and removing both voltage regulators and switches. OpenDSS models these devices as transformers and lines respectively, but with very low impedances.

Five different versions of the IEEE 123-Node System were considered: a base system with (1) distributed generation connected to the bulk transmission grid, (2) distributed synchronous generation for both grid-connected as islanded modes, (3) a single gridforming inverter in islanded mode, (4) multiple grid-forming inverters in islanded mode, and (5) multiple grid-forming and grid-following inverters in islanded mode. The multiple synchronous generators and inverters were installed at the same nodes and with the same power ratings. These nine locations are indicated with red dots in Figure 5, based in part on the modified case study system with distributed generation presented by [73]. Their respective rated powers in MVA are shown in bold fonts. For synchronous generators, the subtransient reactance was assumed to be 0.15 p.u. The centralized inverter is indicated with the bulls-eye symbol, with its power in MVA shown in bold font. Table 2 summarizes the considered network configurations.

Table 2. Summary of case study configurations.

\begin{tabular}{cccc}
\hline Configuration & Generation Type & Generation Placement & Islanding State \\
\hline Base-GC & none & - & grid-connected \\
SG-M-GC & synchronous & multiple & grid-connected \\
SG-M-I & synchronous & multiple & islanded \\
GflI-M-GC & grid-following & multiple & grid-connected \\
GfrI-S-I & grid-forming & multiple & islanded \\
GfrI-M-I & grid-forming & multiple & islanded \\
GflI-GfrI-M-I & grid-following andgrid-forming & islanded \\
\hline
\end{tabular}

In the case of grid-following inverters, OpenDSS contains a PV inverter model with current limiting capability. This was used to validate the introduced grid-following inverter model (Section 2.3). Table 3 provides a comparison of fault currents between the OpenDSS results and the results produced by $\mathrm{PMsP}$ for a single inverter configuration.

In the case of grid-forming inverters, three-phase faults resulted in a system segmented into up to four sections, depending on the exact fault location. To keep the system angles synchronized and help optimization, the inverters at buses $8,48,95$, and 108 were used as reference buses. 
Table 3. Comparison of results for configuration GflI-M-GC of the IEEE 123-Node System.

\begin{tabular}{ccccc}
\hline Fault Node & Fault Phasing & OpenDSS Fault Current (A) & PMsP Fault Current (A) & Difference (\%) \\
\hline 13 & LG & 4385.2 & 4490.6 & +2.40 \\
13 & LL & 5108.1 & 4995.3 & -2.21 \\
13 & 3P & 5315.0 & 5578.8 & +4.96 \\
67 & LG & 2478.3 & 2495.2 & +0.68 \\
67 & LL & 3173.0 & 3132.2 & +1.29 \\
67 & 3P & 3433.5 & 3538.0 & +3.04 \\
113 & LG & 1532.0 & 1545.9 & +0.91 \\
\hline
\end{tabular}

\subsection{Three-Node Islanded Microgrid System}

OpenDSS currently does not support detailed models for grid-forming inverters. To validate the behavior of the introduced grid-forming inverter model (Section 2.4), it was compared against transient simulation results from MATLAB ${ }^{\circledR} /$ Simulink, using the Simscape Electrical Specialized Power Systems acausal blockset [14]. A three-phase inverter with control in a stationary reference frame [74] was implemented based on the model described in [57]. This model is referred to as the "Three-Node Islanded Microgrid System" model and illustrated in Figure 12.

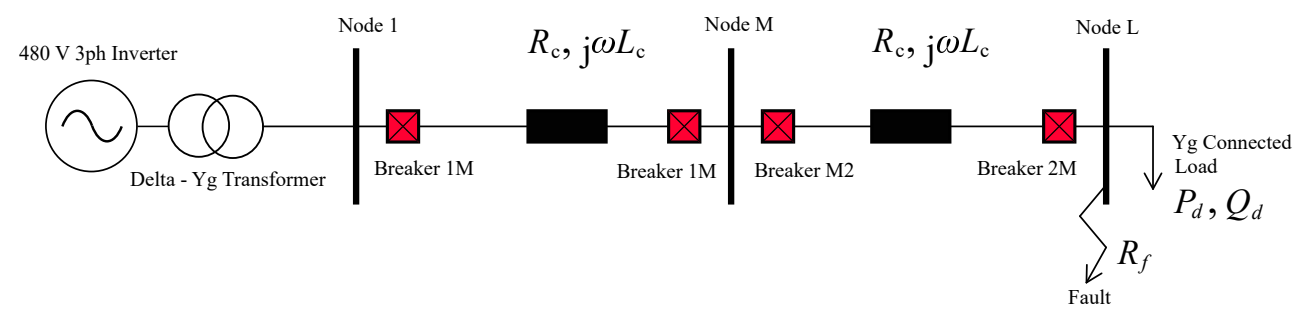

Figure 12. Oneline diagram of the Three-Node Islanded Microgrid System.

Based on [23], a hysteresis approach is applied to limit current during faults. When the root-mean-squared (rms) phase current of the inverter exceeds the hysteresis threshold, the inverter current control loops switch from the output of the voltage control loops to a fixed current reference. Because a three-leg, two-level voltage-source inverter topology is selected, the inverter is not capable of providing zero-sequence current such as needed to supply a ground fault. To overcome this, the inverter is interfaced to the rest of the microgrid with a $480 V_{l l}-480 V_{l n}$ delta-grounded-wye transformer. Tables 4 and 5 lists the parameters of the transient model.

Table 4. Inverter controller parameters.

\begin{tabular}{ccc}
\hline Subsystem & Symbol & Value \\
\hline Voltage loop & $\mathrm{kpv}$ & 0.35 \\
Voltage loop & $\mathrm{krv}$ & 400 \\
Voltage loop & $\mathrm{kvh5}$ & 4 \\
Voltage loop & $\mathrm{kvh7}$ & 20 \\
Voltage loop & $\mathrm{kvh} 11$ & 11 \\
Current loop & $\mathrm{kpi}$ & 0.7 \\
Current loop & $\mathrm{kri}$ & 400 \\
Current loop & $\mathrm{kih5}$ & 30 \\
Current loop & $\mathrm{kih} 7$ & 30 \\
Current loop & $\mathrm{kih} 11$ & 30 \\
\hline
\end{tabular}


Table 5. Hardware parameters.

\begin{tabular}{cccc}
\hline Name & Symbol & Value & Unit \\
\hline Fundamental frequency & $\mathrm{f}$ & 60 & $\mathrm{~Hz}$ \\
Line-line voltage & $\mathrm{V}$ & 480 & $\mathrm{~V}$ \\
Inverter rated power & $\mathrm{P}$ & 50 & $\mathrm{~kW}$ \\
DC-bus voltage & $\mathrm{Vdc}$ & 1800 & $\mathrm{~V}$ \\
Output filter inductance & $\mathrm{L}$ & 18 & $\mu \mathrm{F}$ \\
Output filter capacitance & $\mathrm{C}$ & 250 & $\mathrm{nF}$ \\
Maximum rms output current & $\mathrm{Imax}$ & 70 & $\mathrm{~A}$ \\
Cable resistance & $\mathrm{Rc}$ & 39 & $\mathrm{~m} \Omega$ \\
Cable inductance & $\mathrm{Lc}$ & 70.8 & $\mu \mathrm{H}$ \\
Load real power & $\mathrm{Pd}$ & 25 & $\mathrm{~kW}$ \\
Load reactive power & $\mathrm{Qd}$ & 12.5 & $\mathrm{~kW}$ \\
\hline
\end{tabular}

\section{Results}

Simulation results are provided for both the IEEE 123-Node System and the ThreeNode Islanded Microgrid System. These results are compared with those produced by OpenDSS and MATLAB ${ }^{\circledR} /$ Simulink, respectively.

\subsection{IEEE 123-Node System}

In the case of the Base-GC configuration - that is, grid-connected base network without distributed generation - the short-circuit results of PMsP are presented in Column 3 of Table 6. These results are comparable with those of OpenDSS (see Table 1).

In the case of the SG-M-GC and SG-M-I configurations-that is, grid-connected or islanded operation with multiple distributed synchronous generators-the results are presented in Column 4 and Column 5 of Table 6, respectively. These results are not directly comparable with results from OpenDSS as they are based on a flat start, while OpenDSS performs a pre-fault power flow to determine generator setpoints.

In the case of the GfII-M-GC configuration-that is, grid-connected operation with multiple grid-following inverter-interfaced generators-the short-circuit fault current results of PMsP were within 5\% of the results produced with the PV inverter model of OpenDSS (note that the nominal current is $32.76 \mathrm{~A}$ ). This demonstrated that the formulation of the grid-following inverter constraints (Section 2.3) is able to limit the current injected from the inverter during a fault.

Table 6. Comparison of results for configurations Base-GC, SG-M-GC and SG-M-I of the IEEE 123-Node System.

\begin{tabular}{ccccc}
\hline Fault Node & Fault Phasing & Base-GC Fault Current (A) & SG-M-GC Fault Current (A) & SG- $\boldsymbol{M}$-I Fault Current (A) \\
\hline 13 & LG & 4444.9 & 5914.654 & 3969.269 \\
13 & LL & 4954.2 & 6211.437 & 3914.959 \\
13 & 3P & 5517.3 & 7116.605 & 4559.597 \\
67 & LG & 2362.2 & 4788.279 & 3896.403 \\
67 & LL & 2933.1 & 5301.483 & 3969.117 \\
67 & 3P & 3285.6 & 6153.95 & 4598.621 \\
113 & LG & 1392.2 & 2987.6 & 2802.407 \\
\hline
\end{tabular}

In the case of the GfrI-S-I configuration, a single grid-forming inverter-interfaced generator was placed near the substation at Bus 8 and became the main source of power for the islanded system. When the system experienced a heavy fault, the inverter went into current limiting mode and appeared as a current source, thereby limiting the fault currents to the maximum current that could be injected by the inverter.

In the case of the GfrI-M-I and GflI-GfrI-M-I configurations, multiple grid-forming (and grid-following) inverter-interfaced generators were placed throughout the islanded 
system according to Figure 5. This resulted in the faults being fed from multiple lines connected to the faulted bus. These configurations had fault currents less than the GfrI-S-I islanded configuration (Table 3) or any of the grid-connected configurations (Tables 1, 7, and 8).

It can be concluded that there are two reasons for the lower currents in the multiple inverter configurations of Tables 7 and 8: (1) a difference in the lower capacity of the configurations, and (2) an increase in the electrical distances between the sources and the fault location.

To the first point: The total capacity of the configurations with inverter-interfaced distributed generation was 5.7 MVA, while the single grid-forming inverter had a capacity of 6 MVA. PMsP partially bases the maximum fault current on the VA rating of the inverter, which explains why the 1165 A value seen in Table 8 was close to, but higher than the maximum fault currents in the multiple inverter configurations seen in the same table.

Table 7. Comparison of results for configurations GfrI-S-I, GfrI-M-I, and GflI-GfrI-M-I of the IEEE 123-Node System, and $20 \Omega$ fault resistance.

\begin{tabular}{ccccc}
\hline Fault Node & Fault Phasing & GfrI-S-I Fault Current (A) & GfrI-M-I Fault Current (A) & GflI-GfrI-M-I Fault Current (A) \\
\hline 13 & LG & 119.1 & 99.4 & 114.5 \\
13 & LL & 206.0 & 138.9 & 148.7 \\
13 & SP & 355.4 & 229.0 & 171.9 \\
67 & LG & 118.0 & 73.6 & 107.7 \\
67 & LL & 203.3 & 177.5 & 168.0 \\
67 & 3P & 355.4 & 236.8 & 170.0 \\
113 & LG & 115.2 & 74.4 & 62.7 \\
\hline
\end{tabular}

Table 8. Comparison of results for configurations GfrI-S-I, GfrI-M-I, and GflI-GfrI-M-I of the IEEE 123-Node System, and $20 \Omega$ fault resistance.

\begin{tabular}{ccccc}
\hline Fault Node & Fault Phasing & GfrI-S-I Fault Current (A) & GfrI-M-I Fault Current (A) & GflI-GfrI-M-I Fault Current (A) \\
\hline 13 & LG & 1165.7 & 816.6 & 861.8 \\
13 & LL & 1165.7 & 892.6 & 883.5 \\
13 & 3P & 1165.8 & 1082.1 & 1087.2 \\
67 & LG & 1165.7 & 11023.4 & 1008.8 \\
67 & LL & 1165.7 & 839.7 & 963.6 \\
67 & SP & 1165.8 & 1080.3 & 1091.0 \\
113 & LG & 1165.7 & 446.1 & 475.1 \\
\hline
\end{tabular}

To the second point: The lower currents are also due to the increased electrical distances between the sources and the fault locations. In the grid-connected and single inverter configurations the sources were directly connected to the main trunk of the feeder, whereas in the multiple inverter configurations some of the inverters were placed further down the laterals. This resulted in larger impedances between the inverters and the fault location, thereby reducing the fault current contributions of those inverters.

\subsection{Three-Node Islanded Microgrid System}

Table 9 presents a comparison of measured fault currents between the PMsP gridfollowing inverter model and the transient simulation results produced by MATLAB $^{\circledR} /$ Simulink (using the model of Figure 13). It can be concluded that the fault currents returned by PMsP are higher due to using the delta-grounded-wye transformer model. At the moment, the delta-grounded-wye model for PowerModelsDistribution.j1 [59] is a work in progress. Instead, an approximation of the delta-wye-grounded transformer, used in the Simulink model, was implemented in PMsP for the performed tests, however, it does not model a ground impedance that explains the higher current for the line-to-ground low impedance fault. 


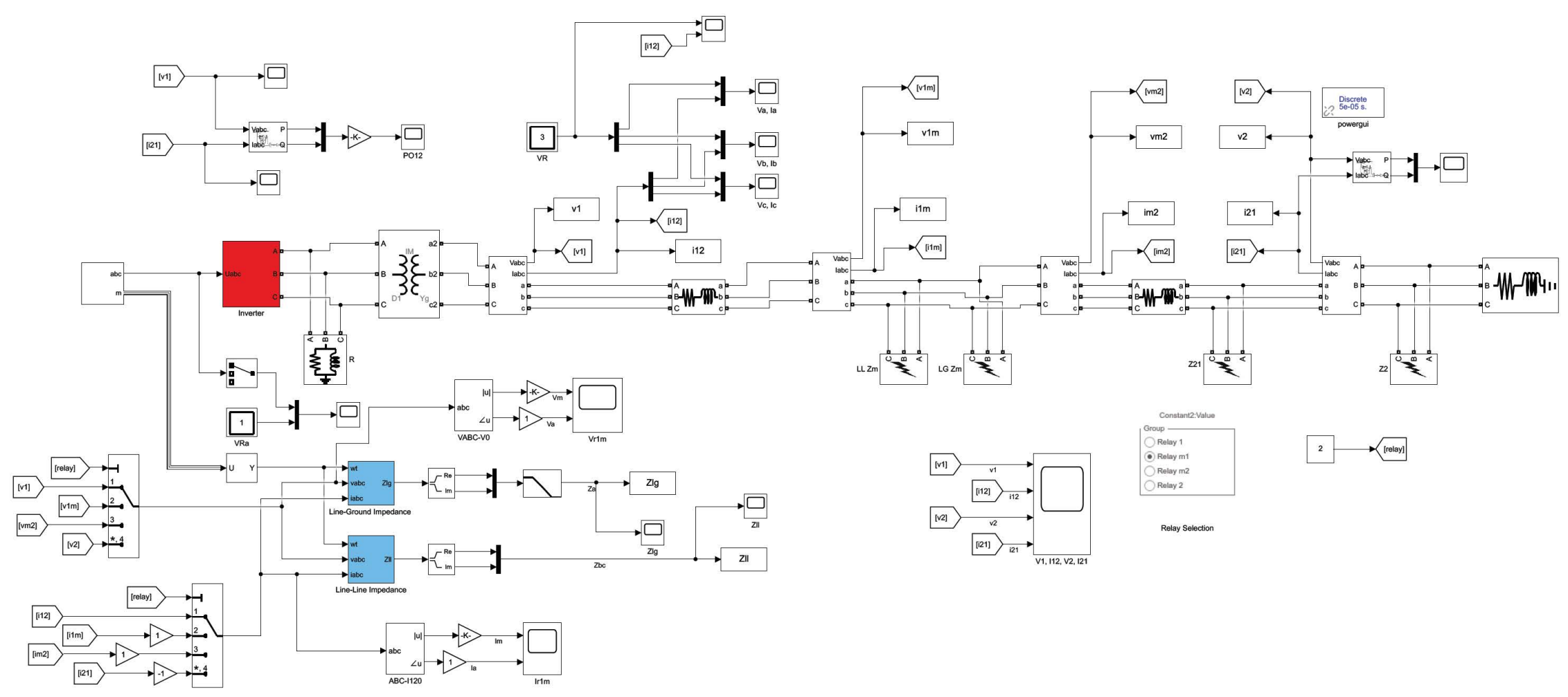

Figure 13. Transient microgrid model. 
Table 9. Comparison of results for a grid-forming inverter on the microgrid of Figure 12.

\begin{tabular}{ccccc}
\hline Fault Resistance $(\boldsymbol{\Omega})$ & Fault Phasing & Simulink Fault Current (A) & PMsP Fault Current (A) & Difference (\%) \\
\hline 20.0 & LG & 13.79 & 13.73 & -0.44 \\
20.0 & LL & 11.94 & 11.89 & -0.42 \\
20.0 & 3P & 13.79 & 13.73 & -0.44 \\
$5.0 \mathrm{~m}$ & LG & 103.4 & 114.6 & -10.8 \\
$5.0 \mathrm{~m}$ & LL & 60.55 & 57.29 & -5.38 \\
$5.0 \mathrm{~m}$ & 3P & 69.93 & 66.15 & -5.41 \\
\hline
\end{tabular}

\section{Conclusions}

This paper investigated the concept of performing short-circuit analysis within an optimization problem formulation. An optimization formulation for short-circuit currents on unbalanced distribution networks was derived and implemented in the PowerModels.jl [58] ecosystem in the Julia programming language. This formulation was expressed as a feasibility problem that explicitly represents voltages and currents during short-circuit conditions. It is based on the rectangular coordinates of the voltages and currents of the model, with a current balancing constraint based on Kirchoff's Current Law coupling the model together and providing an expression for determining the fault current at a particular bus. Results from the IEEE 123-Node System showed that the optimization-based approach was able to produce fault currents within $5 \%$ of the results produced by OpenDSS and the published results of the IEEE Power and Energy Society.

Constraints were developed for the short-circuit contributions of both grid-following and grid-forming inverters. These constraints not only represent the current and power limitations of the inverters, but also the operational constraints based on the inverter type for both normal and faulted conditions. The developed formulation as a whole and the individual inverter short-circuit models were validated against existing models: Experimental validation of the grid-following inverter constraints in PMsP was performed against OpenDSS on the IEEE 123-Node System with added inverter-interfaced generation; reported fault current magnitudes were within 5\% of those calculated by OpenDSS. Experimental validation of the grid-forming inverter constraints in PMsP was performed against a built dynamic simulation model implemented in MATLAB ${ }^{\circledR} /$ Simulink; fault currents calculated by PMsP and Simulink agreed closely on high-impedance faults, however, for the case of low impedance faults, results showed noticeable differences that can be attributed to the present level of detail in transformer modeling under PowerModelsDistribution.j1 [59]. In addition, experiments were performed on the grid-forming inverter in both grid-connected and islanded modes in order to demonstrate the ability of the constraints to conform to the inverters supporting the system voltage or being the main source of voltage in an island.

The use of the constraints presented in this paper in optimization problems has the potential to simplify short-circuit analysis involving inverter-interfaced networked microgrids and to assist optimal operation formulations to select solutions that provide protection coordination. Modeling inverter-interfaced generation involves constraints that are not straightforward to put into a traditional, Thevenin equivalent based short-circuit solver, and furthermore requires nested iterative solution methods. This concept was implemented and demonstrated in PMsP, and tested on the IEEE 123-Node System for both synchronous and inverter-interfaced generations.

Optimization-based short-circuit analysis offers the potential to integrate operational switching problems within the process of verifying protection coordination constraints by introducing a coupled multi-network formulation, similar to that employed by PowerModelsGMD.j1 [61]. Such an approach allows one to perform optimal operational switching problems with switching constraints on larger microgrids, where the number of switch states precludes enumerating the protection-feasible switch-states a priori with a traditional fault study. 
Several opportunities for future work have been identified: First, improving the accuracy of the optimization-based formulation by including the effects of multiple transformer winding configurations, particularly with respect to grounding. Second, developing standardization, as the lack of it means that there are currently a handful of different currentsharing and current-limiting modes for inverters. Third, there is opportunity to investigate the impacts of these inverter models on operation, in terms of contribution to short-circuit currents. Last, while the short-circuit formulation for conventional generation is convex, the constraints for inverter short-circuit contribution are not. Consequently, there is potential for investigating the feasibility of creating convex relaxations and approximations of these constraints.

Author Contributions: Conceptualization, A.K.B., J.E.T., and A.M. Investigation, A.K.B., J.E.T., and A.M. Software, A.K.B. and J.E.T. Validation, A.K.B., J.E.T., and A.M. Writing-original draft preparation, A.K.B., J.E.T., and A.M. Writing-review and editing, A.K.B., A.M., and R.W.B. Visualization, A.K.B. and J.T. Supervision, A.K.B. All authors have read and agreed to the published version of the manuscript.

Funding: This work was performed with the support of the U.S. Department of Energy (DOE) Office of Electricity (OE) Microgrid Research and Development Program under the Resilient Operations of Networked Microgrids (RONM) project.

Acknowledgments: The program manager for the DOE OE program supporting this work is Dan Ton. We gratefully acknowledge Dan's support of this work.

Conflicts of Interest: The authors declare no conflict of interest. The funders had no role in the design of the study; in the collection, analyses, or interpretation of data; in the writing of the manuscript, or in the decision to publish the results.

\section{Appendix A. Three-Phase Power Flow Notation}

Appendix A documents the notation required to define the fundamentals of the mathematical formulations. The notation discussed here is used in the ac unbalanced three-phase power flow. These equations are used as the core power flow formulation dictating the physics of power flow in distribution feeders with networkable microgrids. It should be noted that not all of the following parameters and variables are used in the formulation presented hererin, but are included for a general formulation that could be used in power flow and optimal power flow formulations.

Sets:

$\mathbb{C}$-set of complex numbers

$\mathcal{N}$-set of buses (nodes), indexed by $i$

$\mathcal{E}$ - set of edges (lines and transformers), indexed by $(i, j, k)$; each edge is assigned an arbitrary direction from a bus $i$ to a bus $j$ and is uniquely identified with $k$

$\chi \in \mathcal{E}$ - set of three-phase transformers indexed by $(i, j, k)$

$\mathcal{G}$-set of generator, indexed by $g$

$\mathcal{G}_{i}$ - set of generator connected to bus $i \in \mathcal{N}$, indexed by $g$

$\mathcal{B}$-set of batteries, indexed by $\beta$

$\mathcal{B}_{i}$ - set of batteries connected to bus $i \in \mathcal{N}$, indexed by $\beta$

$\mathcal{E}_{i}^{+}$—set of outgoing edges from $i$, indexed by $(i, j, k)$

$\mathcal{E}_{i}^{-}$- set of incoming edges to $i$, indexed by $(j, i, k)$

$\Phi=$ - phase set

$\Phi_{i} \subseteq\{a, b, c\}$-phase set for bus $i$

$\Phi_{i j k} \subseteq\{a, b, c\}$ - phase set for line $(i, j, k)$

Parameters:

$\boldsymbol{Y}_{i j k}^{\Phi}=\left[\begin{array}{ccc}\boldsymbol{Y}_{i j k}^{a a} & \boldsymbol{Y}_{i j k}^{a b} & \boldsymbol{Y}_{i j k}^{a c} \\ \bullet & \boldsymbol{Y}_{i j k}^{b b} & \boldsymbol{Y}_{i j k}^{b c} \\ \bullet & \bullet & \boldsymbol{Y}_{i j k}^{c c}\end{array}\right] \in \mathbb{C}^{6}$-symmetric complex admittance matrix for edge $(i, j, k) \in E$ 
$Z_{i j k}^{\Phi}=\left(Y_{i j k}^{\Phi}\right)^{-1}$-symmetric complex impedance matrix for edge $(i, j, k) \in E$

$Y_{i j k}^{1}, Y_{i j k}^{2}$-admittance matrix of three-phase transformer $(i, j, k)$

$\boldsymbol{y}_{i j k}$-admittance of transformer $(i, j, k)$

$\eta_{i j k}$-tap ratio of transformer $(i, j, k)$

$W_{i j k}$-connection matrix of three-phase transformer $(i, j, k)$

Variables:

$I_{g}^{\Phi}=\left(\ell_{g}^{\phi}+\mathbf{j} m_{g}^{\phi}, \phi \in \Phi\right) \in \mathbb{C}^{3}$-vector of complex current generation at $g \in \mathcal{G}$

$I_{\beta}^{\Phi}=\left(\ell_{\beta}^{\phi}+\mathbf{j} m_{\beta}^{\phi}, \phi \in \Phi\right) \in \mathbb{C}^{3}$-vector of complex current provided (injected) at $\beta \in \mathcal{B}$

$I_{i}^{\Phi}=\left(l_{i}^{\phi}+\mathbf{j} m_{i}^{\phi}, \phi \in \Phi\right) \in \mathbb{C}^{3}$-vector of complex current consumed at $i \in \mathcal{N}$

$I_{i j k}^{\Phi}=\left(\ell_{i j k}^{\phi}+\mathbf{j} m_{i j k^{\prime}}^{\phi}, \phi \in \Phi\right) \in \mathbb{C}^{3}$-vector of complex current flow on line $(i, j) \in \mathcal{E}$

$V_{i}^{\Phi} \in \mathbb{C}^{3}$-vector of complex voltage on bus $i \in \mathcal{N}$

$S_{g}^{\Phi}=\left(p_{g}^{\phi}+\mathbf{j} q_{g}^{\phi}, \phi \in \Phi\right) \in \mathbb{C}^{3}$-vector of complex power generation at $g \in G$

$S_{\beta}^{\Phi}=\left(p_{\beta}^{\phi}+\mathbf{j} q_{\beta}^{\phi}, \phi \in \Phi\right) \in \mathbb{C}^{3}$-vector of complex power provided (injected) at $\beta \in \mathcal{B}$

$S_{i}^{\Phi}=\left(p_{i}^{\phi}+\mathbf{j} q_{i}^{\phi}, \phi \in \Phi\right) \in \mathbb{C}^{3}$-vector of complex power consumed at $i \in \mathcal{N}$

$S_{i j k}^{\Phi}=\left(p_{i j k}^{\phi}+\mathbf{j} q_{i j k}^{\phi}, \phi \in \Phi\right) \in \mathbb{C}^{3}$-vector of complex power flow on line $(i, j, k) \in \mathcal{E}$

\section{Appendix B. Short-Circuit Formulation Notation}

Appendix $B$ documents the notation required to define the fundamentals of the mathematical formulations. The notation discussed here is used to model short-circuit analysis.

Sets:

$\mathcal{F}$-set of faults, indexed by $f$

$\mathcal{G}_{p q i}$ - set of grid-following inverters, indexed by $g$

$\mathcal{G}_{v i}$ - set of grid-forming inverters, indexed by $g$

\section{Parameters:}

$i_{f}$ - the bus of fault $f$

$\boldsymbol{G}_{f}$-admittance matrix of fault $f$

$\alpha$-complex parameter used for calculating sequence components

$\hat{r}_{g}$-virtual impedance real part upper limit, indexed by $g$

$\hat{x}_{g}$-virtual impedance imaginary part upper limit, indexed by $g$

$\boldsymbol{M}_{g}$-scaling for voltage upper and lower limit, indexed by $g$

$V_{g f}^{\Phi r e f}$-reference voltage of inverter, indexed by $g$

\section{Variables:}

$I_{g f}^{\Phi}=\left(\ell_{g f}^{\phi}+\mathbf{j} m_{g f}^{\phi}, \phi \in \Phi\right) \in \mathbb{C}^{3}$-vector of complex fault current generation at $g \in \mathcal{G}$ for fault $f$

$I_{s i_{f} f}^{\Phi}=\left(\ell_{i_{f} f}^{\phi}+\mathbf{j} m_{i_{f} f}^{\phi}, \phi \in \Phi\right) \in \mathbb{C}^{3}$-vector of complex fault current at $i_{f} \in \mathcal{N}$ for fault $f$

$I_{i f}^{\Phi}=\left(\ell_{i f}^{\phi}+\mathbf{j} m_{i f}^{\phi}, \phi \in \Phi\right) \in \mathbb{C}^{3}$-vector of complex load current at $i \in \mathcal{N}$ for fault $f$

$I_{i j k f}^{\Phi}=\left(\ell_{i j k f}^{\phi}+\mathbf{j} m_{i j k f}^{\phi}, \phi \in \Phi\right) \in \mathbb{C}^{3}$-vector of complex fault current flow on line $(i, j) \in \mathcal{E}$

for fault $f$

$V_{i f}^{\Phi} \in \mathbb{C}^{3}$-vector of complex voltage on bus $i \in \mathcal{N}$ for fault $f$

$r_{g f}^{\Phi}$-virtual resistance of inverter $g$

$x_{g f}^{\Phi}$-virtual reactance inverter $g$

$V_{g f}^{\Phi s e t}$ —voltage set point of inverter $g$ 


\section{Appendix C. Protection Formulation Notation}

Appendix $C$ documents the notation required to define the fundamentals of the mathematical formulations. The notation discussed here is used to model protection.

Sets:

$\mathcal{Z}$-set of protection zones, indexed by $\zeta$

$\mathcal{P}$-set of protective devices, indexed by $p$

$\mathcal{P}_{\zeta}$ - set of protective devices in zone $\zeta$, indexed by $p$

$\mathcal{E}_{\zeta}$ - set of branches with protective devices, indexed by $p$

Parameters:

$\mathcal{Z}_{i j k}^{l g, o p}$ _operating area for ground fault admittance relay $(i, j, k)$

$\mathcal{Z}_{i j k}^{l l, o p}$ _operating area for line fault admittance relay $(i, j, k)$

$A=0.14$-time-overcurrent relay time-dial scaling

$B=0.02$-time-overcurrent relay curve shape

$T_{c}=0.5 \mathrm{~s}$-coordination time interval for time-overcurrent relays

$\hat{t}^{p}=7 \mathrm{~s}-$ maximum allowable trip time for time-overcurrent relays

\section{Variables:}

$T_{i j k}$-time-overcurrent relay time-dial setting, indexed by $(i, j, k)$

$I_{i j k}^{p}$-time-overcurrent relay pickup current, indexed by $(i, j, k)$

$t_{i j k}^{p}$-time-overcurrent relay trip time, indexed by $(i, j, k)$

$t_{(1)}^{p}-1$ st order statistic of time-overcurrent relay trip times

$\tilde{Z}_{i j k f}^{l g+}$-Measured positive-sequence impedance between relay $(i, j, k)$ and fault for lineground faults

$\tilde{Z}_{i j k f}^{l l+}$-Measured positive-sequence impedance between relay $(i, j, k)$ and fault for lineline faults

$\tilde{Z}_{i j k f}^{+}$-Measured positive-sequence impedance at relay $(i, j, k)$

$\tilde{Z}_{i j k f}^{0}$-Measured zero-sequence impedance at relay $(i, j, k)$

$k_{i j k f}$-Residual compensation factor for ground-fault relay $(i, j, k)$

\section{Appendix D. Restoration Notation}

Appendix D documents the notation required to define the fundamentals of the mathematical formulations. The notation discussed here is used to model common engineering limits and constraints, which restrict the operations of distribution feeder restoration with microgrids.

\section{Parameters:}

$S_{i r}^{\Phi}=\left(p_{i r}^{\phi}+\mathbf{j} q_{i r}^{\phi}, \phi \in \Phi\right) \in \mathbb{C}^{3}$-vector of complex power demand at bus $i \in \mathcal{N}$ during restoration

Variables:

$I_{g r}^{\Phi}=\left(\ell_{g r}^{\phi}+\mathbf{j} m_{g r}^{\phi}, \phi \in \Phi\right) \in \mathbb{C}^{3}$-vector of complex current generation at $g \in \mathcal{G}$ during restoration

$I_{i r}^{\Phi}=\left(l_{i r}^{\phi}+\mathbf{j} m_{i r}^{\phi}, \phi \in \Phi\right) \in \mathbb{C}^{3}$-vector of complex current consumed at $i \in \mathcal{N}$ during restoration

$I_{i j k r}^{\Phi}=\left(\ell_{i j k r}^{\phi}+\mathbf{j} m_{i j k r}^{\phi}, \phi \in \Phi\right) \in \mathbb{C}^{3}$-vector of complex current flow on line $(i, j) \in \mathcal{E}$ during restoration

$V_{i r}^{\Phi} \in \mathbb{C}^{3}$-vector of complex voltage on bus $i \in \mathcal{N}$ during restoration

$S_{g r}^{\Phi}=\left(p_{g r}^{\phi}+\mathbf{j} q_{g r}^{\phi}, \phi \in \Phi\right) \in \mathbb{C}^{3}$-vector of complex power generation at $g \in G$ during restoration

$S_{i r}^{\Phi}=\left(p_{i r}^{\phi}+\mathbf{j} q_{i r}^{\phi}, \phi \in \Phi\right) \in \mathbb{C}^{3}$ —vector of complex power consumed at $i \in \mathcal{N}$ during restora- 
tion

$S_{i j k r}^{\Phi}=\left(p_{i j k r}^{\phi}+\mathbf{j} q_{i j k r}^{\phi}, \phi \in \Phi\right) \in \mathbb{C}^{3}$ —vector of complex power flow on line $(i, j, k) \in \mathcal{E}$ during restoration $\delta_{i j k r} \in[0,1]$ 一open and close variable for edge $(i, j, k) \in \Gamma_{t} ; \delta_{i j k r}=0$ denotes an open edge, $\delta_{i j k r}=1$ denotes a closed edge

$\delta_{i r} \in[0,1]$ - on and off variable for the load at bus $i$ during restoration; $\delta_{i r}=0$ denotes the load is not energized, $\delta_{i r}=1$ denotes the load is energized

$\delta_{g r} \in[0,1]$ - on and off variable for the generator $g$ during restoration; $\delta_{g r}=0$ denotes the generator is off, $\delta_{g r}=1$ denotes the generator is on

\section{References}

1. Alam, M.N.; Chakrabarti, S.; Ghosh, A. Networked Microgrids: State-of-the-Art and Future Perspectives. IEEE Trans. Ind. Inform. 2019, 15, 1238-1250. [CrossRef]

2. Venkata, S.S.; Reno, M.J.; Bower, W.; Manson, S.; Reilly, J.; Sey, G.W., Jr. Microgrid Protection: Advancing the State-of-the-Art; Tech Report; SAND2019-3167; Sandia National Laboratories: Albuquerque, NM, USA, 2019.

3. McDermott, T.; Vyakaranam, B.; Fan, R.; Mana, P.T.; Smith, T.; Li, Z.; Hambrick, J.; Barnes, A.K. Protective Relaying for Distribution and Microgrids Evolving from Radial to Bi-Directional Power Flow. In Proceedings of the 2018 Western Protective Relay Conference, Spokane, WA, USA, 15-18 October 2018.

4. Tumilty, R.M.; Brucoli, M.; Burt, G.M.; Green, T.C. Approaches to Network Protection for Inverter Dominated Electrical Distribution Systems. In Proceedings of the 3rd IET International Conference on Power Electronics, Machines and Drives, Dublin, Ireland, 4-6 April 2006; pp. 622-626. [CrossRef]

5. Dewadasa, J.M.; Ghosh, A.; Ledwich, G. Line Protection in Inverter Supplied Networks. In Proceedings of the 2008 Australasian Universities Power Engineering Conference, Sydney, Australia, 14-17 December 2008; pp. 1-6.

6. Lazarou, S.; Vita, V.; Ekonomou, L. Protection Schemes of Meshed Distribution Networks for Smart Grids and Electric Vehicles. Energies 2018, 11, 3106. [CrossRef]

7. Dewadasa, J.M.; Ghosh, A.; Ledwich, G. Distance Protection Solution for a Converter Controlled Microgrid. In Proceedings of the 15th National Power Systems Conference, Mumbai, India, 16-18 December 2008.

8. Kar, S.; Samantaray, S.R. Time-Frequency Transform-Based Differential Scheme for Microgrid Protection. IEEE IET Gener. Transm. Distrib. 2014, 8, 310-320. [CrossRef]

9. Singh, M.; Vishnuvardhan, T.; Srivani, S. Adaptive Protection Coordination Scheme for Power Networks Under Penetration of Distributed Energy Resources. IET Gener. Transm. Distrib. 2016, 10, 3919-3929. [CrossRef]

10. Pavlatos, C.; Vita, V. Linguistic Representation of Power System Signals; Springer: Berlin/Heidelberg, Germany, 2016; pp. 285-295. [CrossRef]

11. Jamali, S.; Borhani-Bahabadi, H. Non-Communication Protection Method for Meshed and Radial Distribution Networks With Synchronous-Based DG. Int. J. Electr. Power Energy Syst. 2017, 93, 468-478. [CrossRef]

12. Barnes, A.K.; Mate, A. Implementing Admittance Relaying for Microgrid Protection. In Proceedings of the 2021 IEEE/IAS 57th Industrial and Commercial Power Systems Technical Conference, Las Vegas, NV, USA, 27-30 April 2021; LA-UR-20-30128; pp. 1-9.

13. Barnes, A.K.; Mate, A. Dynamic State Estimation for Radial Microgrid Protection. In Proceedings of the 2021 IEEE/IAS 57th Industrial and Commercial Power Systems Technical Conference,Las Vegas, NV, USA, 27-30 April 2021; LA-UR-20-30126; pp. 1-9.

14. MathWorks. MATLAB ${ }^{\circledR}$ Simscape. Available online: https://www.mathworks.com/products/simscape.html (accessed on 9 April 2021).

15. Powersim Inc. PSIM. Available online: https://powersimtech.com/products/psim (accessed on 9 April 2021).

16. Plexim. PLECS ${ }^{\circledR}$. Available online: https://www.plexim.com/products/plecs (accessed on 9 April 2021).

17. Manitoba Hydro International Ltd. PSCAD. Available online: https://www.pscad.com (accessed on 9 April 2021).

18. EMTP Alliance. EMTP ${ }^{\circledR}$. Available online: https://www.emtp.com (accessed on 9 April 2021).

19. European EMTP-ATP Group. ATP-EMTP. Available online: https://www.emtp.org (accessed on 9 April 2021).

20. Blackburn, J.L. Protective Relaying Principles and Applications, 3rd ed.; CRC Press: Boca Raton, FL, USA, 2007.

21. Pan, Y.; Ren, W.; Ray, S.; Walling, R.; Reichard, M. Impact of Inverter Interfaced Distributed Generation on Overcurrent Protection in Distribution Systems. In Proceedings of the 2011 IEEE Power Engineering and Automation Conference, Wuhan, China, 8-9 September 2011; pp. 1-6. [CrossRef]

22. Behnke, M.; Ellis, A. Contribution of Photovoltaic Power Generation Systems to AC Short Circuits-A Survey of Current Modeling Practices and Challenges. In Proceedings of the 2013 IEEE 39th Photovoltaic Specialists Conference, Tampa, FL, USA, 16-21 June 2013; pp. 3128-3133. [CrossRef]

23. Bottrell, N.; Green, T.C. Comparison of Current-Limiting Strategies During Fault Ride-Through of Inverters to Prevent Latch-Up and Wind-Up. IEEE Trans. Power Electron. 2014, 29, 3786-3797. [CrossRef]

24. Cui, C.; Li, S. A Microgrid Protection Scheme with Conventional Relay Measurements. In Proceedings of the 2018 IEEE Power \& Energy Society General Meeting, Portland, OR, USA, 5-10 August 2018; pp. 1-5. [CrossRef] 
25. Xu, Y.; Mu, L.; Zhang, F.; Mou, Z.; Zhu, J. Analysis of the Fault Characteristics of Microgrid with Inverter-Interfaced Distributed Generators. In Proceedings of the 2019 IEEE 8th International Conference on Advanced Power System Automation and Protection, Xi'an, China, 21-24 October 2019; pp. 1-5. [CrossRef]

26. Najy, W.K.A.; Zeineldin, H.H.; Woon, W.L. Optimal Protection Coordination for Microgrids with Grid-Connected and Islanded Capability. IEEE Trans. Ind. Electron. 2013, 60, 1668-1677. [CrossRef]

27. Singh, M.; Panigrahi, B.K.; Abhyankar, A.R.; Mukherjee, R.; Kundu, R. Optimal Location, Size and Protection Coordination of Distributed Generation in Distribution Network. In Proceedings of the 2013 IEEE Symposium on Swarm Intelligence, Singapore, 16-19 April 2013; pp. 1-7. [CrossRef]

28. Zeineldin, H.H.; Sharaf, H.M.; Ibrahim, D.K.; El-Zahab, E.E.D.A. Optimal Protection Coordination for Meshed Distribution Systems With DG Using Dual Setting Directional Over-Current Relays. IEEE Trans. Smart Grid 2015, 6, 115-123. [CrossRef]

29. Saleh, K.A.; Zeineldin, H.H.; El-Saadany, E.F. Optimal Protection Coordination for Microgrids Considering N-1 Contingency. IEEE Trans. Ind. Inform. 2017, 13, 2270-2278. [CrossRef]

30. Aghdam, T.S.; Karegar, H.K.; Zeineldin, H.H. Transient Stability Constrained Protection Coordination for Distribution Systems With DG. IEEE Trans. Smart Grid 2018, 9, 5733-5741. [CrossRef]

31. Javadi, H.; Mousavi, S.M.A.; Khederzadeh, M. A Novel Approach to Increase FCL Application in Preservation of Over-Current Relays Coordination in Presence of Asynchronous DGs. Int. J. Electr. Power Energy Syst. 2013, 44, 810-815. [CrossRef]

32. Meliopoulos, A.P.S.; Cokkinides, G.J.; Tan, Z.; Choi, S.; Lee, Y.; Myrda, P. Setting-Less Protection: Feasibility Study. In Proceedings of the 2013 46th Hawaii International Conference on System Sciences, Wailea, HI, USA, 7-10 January 2013; pp. $2345-2353$. [CrossRef]

33. Davydova, N.; Shchetinin, D.; Hug, G. Optimization-Based Settingless Algorithm Combining Protection and Fault Identification. IEEE Trans. Power Deliv. 2020, 35, 2136-2148. [CrossRef]

34. Vovos, P.N.; Bialek, J.W. Direct Incorporation of Fault Level Constraints in Optimal Power Flow as a Tool for Network Capacity Analysis. IEEE Trans. Power Syst. 2005, 20, 2125-2134. [CrossRef]

35. Vovos, P.N.; Bialek, J.W. Impact of Fault Level Constraints on the Economic Operation of Power Systems. IEEE Trans. Power Syst. 2006, 21, 1600-1607. [CrossRef]

36. Keane, A.; O'Malley, M. Optimal Allocation of Embedded Generation on the Irish Distribution Network. In Proceedings of the CIRED 2005-18th International Conference and Exhibition on Electricity Distribution, Turin, Italy, 6-9 June 2005; pp. 1-5. [CrossRef]

37. Vovos, P.N.; Song, H.; Cho, K.; Kim, T. A Network Reconfiguration Algorithm for the Reduction of Expected Fault Currents. In Proceedings of the 2013 IEEE Power \& Energy Society General Meeting, Vancouver, BC, Canada, 21-25 July 2013; pp. 1-5. [CrossRef]

38. Moon, G.; Wi, Y.; Lee, K.; Joo, S. Fault Current Constrained Decentralized Optimal Power Flow Incorporating Superconducting Fault Current Limiter. IEEE Trans. Appl. Supercond. 2011, 21, 2157-2160. [CrossRef]

39. Khazali, A.H.; Kalantar, M. Optimal Power Flow Considering Fault Current Level Constraints and Fault Current Limiters. Int. J. Electr. Power Energy Syst. 2014, 59, 204-213. [CrossRef]

40. Ngoc, P.T.; Singh, J.G. Short Circuit Current Level Reduction in Power System by Optimal Placement of Fault Current Limiter. Int. Trans. Electr. Energy Syst. 2017, 27, 1-16. [CrossRef]

41. Moon, G.; Lee, J.; Joo, S. Integrated Generation Capacity and Transmission Network Expansion Planning With Superconducting Fault Current Limiter (SFCL). IEEE Trans. Appl. Supercond. 2013, 23, 1-5. [CrossRef]

42. Teimourzadeh, S.; Aminifar, F. MILP Formulation for Transmission Expansion Planning With Short-Circuit Level Constraints. IEEE Trans. Power Syst. 2016, 31, 3109-3118. [CrossRef]

43. Lee, S.; Kim, H.; Kim, T.H.; Shin, H.; Kim, W. Fault Current Constraint Transmission Expansion Planning Based on the Inverse Matrix Modification Lemma and a Valid Inequality. Energies 2019, 12, 4769. [CrossRef]

44. Hernandez-Alvidrez, J.; Summers, A.; Reno, M.; Flicker, J.; Pragallapati, N. Simulation of Grid-Forming Inverters Dynamic Models using a Power Hardware-in-the-Loop Testbed. In Proceedings of the 2019 IEEE 46th Photovoltaic Specialists Conference, Chicago, IL, USA, 16-21 June 2019; pp. 1-8. [CrossRef]

45. Pei, X.; Kang, Y. Short-Circuit Fault Protection Strategy for High-Power Three-Phase Three-Wire Inverter. IEEE Trans. Ind. Inform. 2012, 8, 545-553. [CrossRef]

46. Gonzalez, S.; Gurule, N.; Reno, M.J.; Johnson, J. Fault Current Experimental Results of Photovoltaic Inverters Operating with Grid-Support Functionality. In Proceedings of the 2018 IEEE 7th World Conference on Photovoltaic Energy Conversion, Waikoloa, HI, USA, 10-15 June 2018; pp. 1406-1411. [CrossRef]

47. Gurule, N.S.; Hernandez-Alvidrez, J.; Reno, M.J.; Summers, A.; Gonzalez, S.; Flicker, J. Grid-forming Inverter Experimental Testing of Fault Current Contributions. In Proceedings of the 2019 IEEE 46th Photovoltaic Specialists Conference, Chicago, IL, USA, 16-21 June 2019; pp. 1-6. [CrossRef]

48. Kou, G.; Chen, L.; VanSant, P.; Velez-Cedeno, F.; Liu, Y. Fault Characteristics of Distributed Solar Generation. IEEE Trans. Power Deliv. 2020, 35, 1062-1064. [CrossRef]

49. Plet, C.A.; Brucoli, M.; McDonald, J.D.F.; Green, T.C. Fault Models of Inverter-Interfaced Distributed Generators: Experimental Verification and Application to Fault Analysis. In Proceedings of the 2011 IEEE Power and Energy Society General Meeting, Detroit, MI, USA, 24-28 July 2011; pp. 1-8. [CrossRef] 
50. Muljadi, E.; Singh, M.; Bravo, R.; Gevorgian, V. Dynamic Model Validation of PV Inverters under Short-Circuit Conditions. In Proceedings of the 2013 IEEE Green Technologies Conference, Denver, CO, USA, 4-5 April 2013; pp. 98-104. [CrossRef]

51. Kauffmann, T.; Karaagac, U.; Kocar, I.; Gras, H.; Mahseredjian, J.; Cetindag, B.; Farantatos, E. Phasor Domain Modeling of Type III Wind Turbine Generator for Protection Studies. In Proceedings of the 2015 IEEE Power Energy Society General Meeting, Denver, CO, USA, 26-30 July 2015; pp. 1-5. [CrossRef]

52. Alfieri, L.; Carpinelli, G.; Bracale, A.; Caramia, P.; Di Fazio, A.R. Impact of Photovoltaic Generators on the Three Phase Short Circuit Operating Conditions. In Proceedings of the 2018 IEEE International Conference on Environment and Electrical Engineering and 2018 IEEE Industrial and Commercial Power Systems Europe, Palermo, Italy, 12-15 June 2018; pp. 1-7. [CrossRef]

53. Carpinelli, G.; Bracale, A.; Caramia, P.; Di Fazio, A.R. Three-Phase Photovoltaic Generators Modeling in Unbalanced Short-Circuit Operating Conditions. Int. J. Electr. Power Energy Syst. 2019, 113, 941-951. [CrossRef]

54. Ghanaatian, M.; Lotfifard, S. Sparsity-Based Short-Circuit Analysis of Power Distribution Systems With Inverter Interfaced Distributed Generators. IEEE Trans. Power Syst. 2019, 34, 4857-4868. [CrossRef]

55. Liu, S.; Bi, T.; Liu, Y. Theoretical Analysis on the Short-Circuit Current of Inverter-Interfaced Renewable Energy Generators with Fault-Ride-Through Capability. Sustainability 2017, 10, 44. [CrossRef]

56. Kim, I. Short-Circuit Analysis Models for Unbalanced Inverter-Based Distributed Generation Sources and Loads. IEEE Trans. Power Syst. 2019, 34, 3515-3526. [CrossRef]

57. Vasquez, J.C.; Guerrero, J.M.; Savaghebi, M.; Eloy-Garcia, J.; Teodorescu, R. . Modeling, Analysis, and Design of StationaryReference-Frame Droop-Controlled Parallel Three-Phase Voltage Source Inverters. IEEE Trans. Ind. Electron. 2013, 60, 1271-1280. [CrossRef]

58. Coffrin, C.; Bent, R.; Sundar, K.; Ng, Y.; Lubin, M. PowerModels.jl: An Open-Source Framework for Exploring Power Flow Formulations. In Proceedings of the 2018 Power Systems Computation Conference, Dublin, Ireland, 11-15 June 2018; pp. 1-8. [CrossRef]

59. Fobes, D.M.; Claeys, S.; Geth, F.; Coffrin, C. PowerModelsDistribution.jl: An Open-Source Framework for Exploring Distribution Power Flow Formulations. Electr. Power Syst. Res. 2020, 189, 1-7. [CrossRef]

60. Rhodes, N.; Fobes, D.; Coffrin, C.; Roald, L. PowerModelsRestoration.jl: An Open-Source Framework for Exploring Power Network Restoration Algorithms. Electr. Power Syst. Res. 2021, 190, 1-8. [CrossRef]

61. Mate, A.; Barnes, A.K.; Bent, R.W.; Cotilla-Sanchez, E. Analyzing and Mitigating the Impacts of GMD and EMP Events on the Electrical Grid with PowerModelsGMD.jl. LA-UR-19-29623. arXiv 2021, arXiv:2101.05042.

62. Bezanson, J.; Karpinski, S.; Shah, V.B.; Edelman, A. Julia: A Fast Dynamic Language for Technical Computing. arXiv 2012, arXiv:1209.5145. Available online: https://julialang.org/ (accessed on 9 April 2021).

63. Short, T. OpenDSS. Available online: https://github.com/tshort/OpenDSS (accessed on 9 April 2021).

64. Bent, R.; Barnes, A.; Fobes, D.; Gopinath, S.; Hijazi, H.; Matthews, R.; Nagarajan, H.; Reno, M.; Tabarez, J.; Yang, H. Resilient Operations of Networked Microgrids Formulation; Report; Los Alamos National Laboratory and Sandia National Laboratory: Los Alamos, NM, USA, 2020.

65. IEEE PES AMPS DSAS Test Feeder Working Group. Test Feeder Cases. Available online: https://site.ieee.org/pes-testfeeders/ resources / (accessed on 9 April 2021).

66. Kersting, W.H. Radial Distribution Test Feeders. IEEE Trans. Power Syst. 1991, 6, 975-985. [CrossRef]

67. Mason, C.R. The Art E Science of Protective Relaying, 1st ed.; GE Grid Solutions: Boston, MA, USA, 1956.

68. Pattabiraman, D.; Lasseter, R.H.; Jahns, T.M. Comparison of Grid Following and Grid Forming Control for a High Inverter Penetration Power System. In Proceedings of the 2018 IEEE Power \& Energy Society General Meeting, Portland, OR, USA, 5-9 August 2018; pp. 1-5. [CrossRef]

69. Du, W.; Tuffner, F.; Schneider, K.P.; Lasseter, R.H.; Xie, J.; Chen, Z.; Bhattarai, B.P. Modeling of Grid-Forming and Grid-Following Inverters for Dynamic Simulation of Large-Scale Distribution Systems. IEEE Trans. Power Deliv. 2020, 1-11. [CrossRef]

70. Rocabert, J.; Luna, A.; Blaabjerg, F.; Rodríguez, P. Control of Power Converters in AC Microgrids. IEEE Trans. Power Electron. 2012, 27, 4734-4749. [CrossRef]

71. Liserre, M.; Blaabjerg, F.; Dell'Aquila, A. Step-by-Step Design Procedure for a Grid-Connected Three-Phase PWM Voltage Source Converter. Int. J. Electron. 2004, 91, 445-460. [CrossRef]

72. Gonen, T. Electric Power Distribution Engineering, 3rd ed.; CRC Press: Boca Raton, FL, USA, 2016.

73. Reimer, B.; Khalili, T.; Bidram, A.; Reno, M.J.; Matthews, R.C. Optimal Protection Relay Placement in Microgrids. In Proceedings of the 2020 IEEE Kansas Power and Energy Conference, Manhattan, KS, USA, 13-14 July 2020; pp. 1-6. [CrossRef]

74. Teodorescu, R.; Blaabjerg, F.; Liserre, M. Proportional-Resonant Controllers. A New Breed of Controllers Suitable for GridConnected Voltage-Source Converters. Available online: https://vbn.aau.dk/en/publications/proportional-resonant-controllersa-new-breed-of-controllers-suit (accessed on 11 April 2021). 